\title{
Reversal of the Direction of Rectification Induced by Fermi Level Pinning at Molecule-Electrode Interfaces in Redox-Active Tunneling Junctions
}

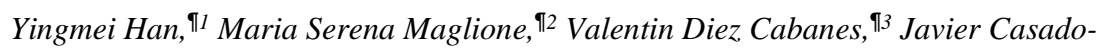

Montenegro, ${ }^{2}$ Xiaojiang Yu, ${ }^{4}$ Senthil Kumar Karuppannan, ${ }^{1}$ Ziyu Zhang, ${ }^{1}$ Núria

Crivillers, ${ }^{2}$ Marta Mas-Torrent, ${ }^{2}$ Concepió Rovira, ${ }^{2}$ Jérôme Cornil,,3* Jaume

Veciana $^{2 *}$ and Christian A. Nijhuis ${ }^{1,5^{*}}$

${ }^{1}$ Department of Chemistry, National University of Singapore, 3 Science Drive 3,

Singapore 117543, Singapore.

${ }^{2}$ Institut de Ciència de Materials de Barcelona (ICMAB-CSIC)/CIBER-BBN, Campus de la UAB, 08193 Bellaterra, Spain.

${ }^{3}$ Laboratory for Chemistry of Novel Materials, University of Mons, Place du Parc 20, 7000 Mons, Belgium.

${ }^{4}$ Singapore Synchrotron Light Source, National University of Singapore, 5 Research Link, Singapore 117603, Singapore.

${ }^{5}$ Centre for Advanced 2D Materials and Graphene Research Center, National University of Singapore, 6 Science Drive 2, Singapore 117546, Singapore.

"Authors contributed equally

*Authors to whom correspondence should be addressed: jerome.cornil@umons.ac.be, vecianaj@icmab.es, and chmnca@nus.edu.sg. 
Abstract: Control over the energy level alignment in molecular junctions is notoriously difficult making it challenging to control basic electronic functions such as the direction of rectification. Therefore, alternative approaches to control electronic functions in molecular junctions are needed. This paper describes switching of the direction of rectification by changing the bottom electrode material $\mathrm{M}=\mathrm{Ag}, \mathrm{Au}$, or $\mathrm{Pt}$ in $\mathrm{M}-\mathrm{S}\left(\mathrm{CH}_{2}\right)_{11} \mathrm{~S}-\mathrm{BTTF} / / \mathrm{EG}$ aIn junctions based on self-assembled monolayers incorporating benzotetrathiafulvalene (BTTF) with EGaIn (eutectic alloy of Ga and In) as the top electrode. The stability of the junctions is determined by the choice of the bottom electrode, which in turn determines the maximum applied bias window, and mechanism of rectification is dominated by the energy levels centered on the BTTF units. The energy level alignment of the 3 junctions are similar due to Fermi level pinning induced by charge transfer at the metal-thiolate interface and by a varying degree of additional charge transfer between BTTF and the metal. Density functional theory (DFT) calculations show that the amount of electron transfer from M to the lowest unoccupied orbital (LUMO) of BTTF follows the order Ag $>\mathrm{Au}>\mathrm{Pt}$. Junctions with Ag electrodes are the least stable and can only withstand an applied bias of $\pm 1.0 \mathrm{~V}$. As a result, no molecular orbitals can fall in the applied bias window and the junctions do not rectify. The junction stability increases for $\mathrm{M}=\mathrm{Au}$ and the highest occupied molecular orbital (HOMO) dominates charge transport at positive bias resulting in a positive rectification ratio of 83 at $\pm 1.5 \mathrm{~V}$. The junctions are very stable for $\mathrm{M}=$ Pt but now the LUMO dominates charge transport at negative bias resulting in a negative rectification ratio of 912 at $\pm 2.5 \mathrm{~V}$. Thus, the limitations of 
Fermi level pinning can be bypassed by a judicious choice of the bottom-electrode material making it possible to access selectively HOMO or LUMO based assisted charge transport and, as shown here, associated reversal of rectification.

Keywords: molecular diodes, molecular electronics, charge transfer, energy level alignment, Fermi-level pinning.

\section{TOC Figure:}

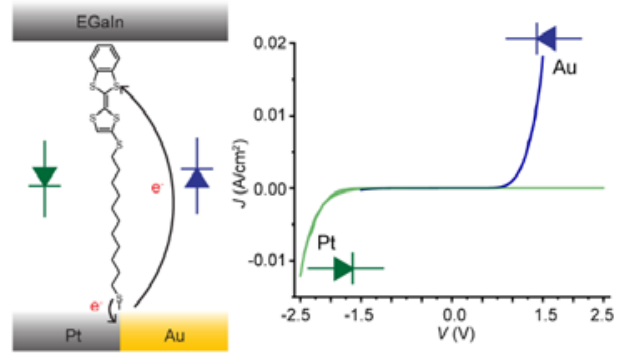


The mechanisms of charge transport and transfer are fundamental to understanding various areas of research ranging from catalysis ${ }^{1}$, biology ${ }^{2}$, to energy management ${ }^{3}$. Molecular tunnel junctions make it possible to uncover and study new phenomena, such as light-matter interactions in tunnel junctions ${ }^{4-7}$, conductance switching ${ }^{8-9}$, molecular sensing ${ }^{10}$ or new mechanisms of charge transport (such as long-range tunneling ${ }^{11-12}$, charge transport in the inverted Marcus region ${ }^{13-15}$, or nuclear tunneling $\left.{ }^{16-19}\right)$, at the molecular length-scales. It is still challenging to control the energy level alignment within molecular junctions as it depends on several intertwined factors related to molecule-electrode interactions involving push back effects $^{20-21}$, Fermi level $\left(E_{\mathrm{f}}\right)$ pinning ${ }^{22-23}$, (partial) charge transfer between the molecule and the

11 surface ${ }^{20,24}$, the formation of surface dipoles and the role of molecular dipoles ${ }^{25-26}$, or collective

12 electrostatic effects ${ }^{27-28}$, complicating the rational design of molecular junctions. For these reasons, it is challenging to control basic electronic functions of molecular junctions, such as, the

14 direction of rectification. Molecular rectification has been one of the hallmarks in molecular electronics since the landmark report by Aviram and Ratner which outlines the theoretical basis of a molecular diode $^{29}$. Although well-performing molecular diodes based on, e.g., donor-acceptor (D-A) compounds ${ }^{13}$, 30-31, large molecular dipoles ${ }^{32-33}$, different interface dipoles ${ }^{34}$, or redox-units ${ }^{35-36}$, have been reported, control over the direction of rectification is limited. The direction of rectification is defined as the bias at which the molecular diodes allows the current to pass

21 through the junctions given by:

$$
\begin{aligned}
& R^{+}=J(+V) / J(-V) \\
& R^{-}=J(-V) / J(+V)
\end{aligned}
$$


where $J$ is the current density that flows across the junctions as a function of the applied voltage

$2 V$. Here, $R^{+}$is the rectification ratio for a junction that allows the current to pass through at

3 positive $V$, but blocks the current at negative $V$, and $R^{-}$defines the same but for a junction with

4 reversed rectification. So far, only a few examples have been reported where reversal of

5 rectification has been demonstrated. In large area junctions, the redox-group is typically located

6 at the top of the monolayer and is therefore in close proximity to the top electrode. Reversal of

7 rectification has been achieved in such systems by moving the redox unit to the bottom of the

8 monolayer ${ }^{37-39}$. Similarly, reversal of rectification has been achieved by moving a dipole from

9 the top to the bottom of the monolayer ${ }^{32}$. In junctions with D-A compounds, changing the orientation of the D-A unit with respect to the electrodes also results in reversal of rectification ${ }^{30}$, ${ }^{40}$. All these strategies require modification of the chemical structure of the molecules via elaborate chemical synthesis. In contrast, here we report reversal of rectification by simply changing the bottom-electrode material.

The energy level alignment at metal—molecule interfaces involves the formation of interface dipoles $(\Delta)$ due to push back effects (molecular adsorption on the metal, which "pushes" back the electron into the metal), which systematically lowers the work function of the metal electrode $(\Phi)$ (although the opposite effect may occur when, for instance, back-bonding

18 from the metal dominates ${ }^{23-24,41}$ ). Often, charge transfer between the molecule and metal surface has also to be considered since it is affecting the interface dipole and may increase or lower $\Phi^{21}$, 42-43. For instance, in alkanethiolate SAMs on coinage metals, charge transfer occurs between the metal and the thiolate and results in a well-known Fermi-level pinning due to the strong interfacial electronic coupling, implying that the work function of the metal after molecular adsorption is independent of the work function of the native metal. In principle, when redox- 
active units are present, charge transfer between the metal surface and the highest occupied molecular orbital (HOMO) or lowest unoccupied molecular orbital (LUMO) centered on the redox units may also occur.

Due to the Fermi level pinning effects, one would expect that the mechanism of charge transport is independent of M. Whitesides and co-workers showed that the mechanism of electrical failure of molecular junctions is independent of the molecular structure, ${ }^{44}$ and we have recently shown that the breakdown mechanism is dominated by direct transfer of momentum of the conduction electrons to the atoms in the electrodes via the wind force resulting in metal filament formation ${ }^{45}$. These studies indicated that the stability of the junctions can be improved with judiciously choses bottom electrode materials. Therefore, by changing the electrode material of the junctions, it should be possible to increase the bias window and access different charge transport regimes and behaviors ${ }^{36,46-47}$. This paper describes reversal of the direction of rectification of molecular diodes of the form $\mathrm{M}-\mathrm{S}\left(\mathrm{CH}_{2}\right)_{11} \mathrm{~S}-\mathrm{BTTF} / / \mathrm{GaO}_{\mathrm{x}} / \mathrm{EGaIn}(\mathrm{BTTF}=$ benzotetrathiafulvalene, EGaIn = eutectic alloy of $\mathrm{Ga}$ and $\mathrm{In}$, and $\mathrm{M}=\mathrm{Ag}, \mathrm{Au}$, or Pt) depending on the choice of the bottom electrode material M. Figure 1 shows schematic illustrations of the junctions and indicates the partial charge transfer from the metal to the BTTF units that is made possible by a super exchange mechanism ${ }^{48-50}$; this partial charge transfer is large for $\mathrm{M}=\mathrm{Ag}$, moderate for $\mathrm{M}=\mathrm{Au}$, and negligible for $\mathrm{M}=\mathrm{Pt}$ resulting in a similar energy level alignment for the three bottom electrodes. Junctions with Ag electrodes can only withstand a narrow bias window of $\pm 1.0 \mathrm{~V}$ and do not rectify. Junctions with $\mathrm{M}=$ Au are more stable with a rectification ratio $R^{+}=83$ at $\pm 1.5 \mathrm{~V}$ involving the HOMO. In contrast, junctions with $\mathrm{M}=\mathrm{Pt}$ are very stable and the current flows across the junction at negative bias with $R^{-}=912$ at $\pm 2.5 \mathrm{~V}$ by involving 
1 the LUMO. These results show that it is possible to control the direction of rectification, despite

2 Fermi level pinning, by changing the bottom-electrode material.

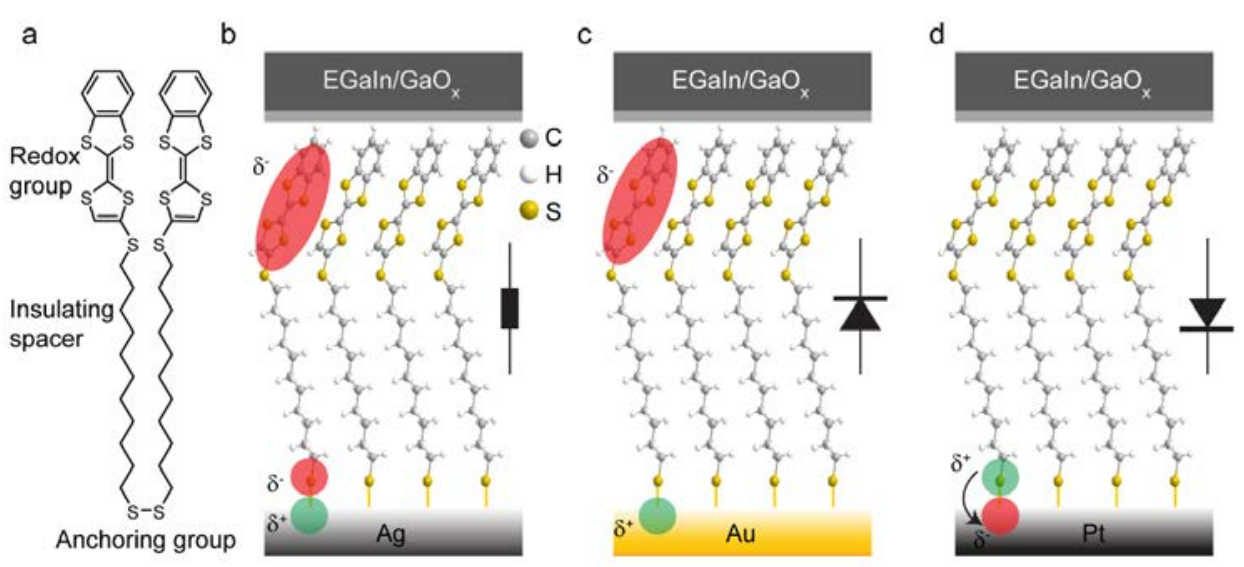

4 Figure 1. (a) Molecular structure of $\left(\mathrm{S}\left(\mathrm{CH}_{2}\right)_{11} \mathrm{~S}-\mathrm{BTTF}\right)_{2}$. (b)-(d) Schematic illustrations of the

$5 \mathrm{M}-\mathrm{S}\left(\mathrm{CH}_{2}\right)_{11} \mathrm{~S}-\mathrm{BTTF} / / \mathrm{GaO}_{\mathrm{x}} / \mathrm{EGaIn}$ junctions with $\mathrm{M}=\mathrm{Ag}, \mathrm{Au}$, and Pt. Red and green ovals

6 indicate negative and positive charges after partial charge transfer as a result of energy level

7 alignment, respectively. The circuit symbols indicate the direction of rectification, or when the

8 junction does not rectify (and behaves as a resistor). $\delta^{+}$and $\delta^{-}$indicate partial charge transfer

9 between the S and BTTF units and the bottom electrode surface as determined from DFT

10 calculations (see Figure 4).

\section{Results and Discussion}

Structural characterization of BTTF SAMs. The synthesis and characterization of the

$\left(\mathrm{S}\left(\mathrm{CH}_{2}\right)_{11} \mathrm{~S}-\mathrm{BTTF}\right)_{2} \mathrm{SAM}$ precursor is described in detail in Section S1. The SAMs were formed on template-stripped $\mathrm{Ag}, \mathrm{Au}$, and Pt (Despite that the three types of surfaces have similar surface roughnesses, the surface topography of the three metal surfaces is different with the Ag surface 

are very small. We have reported before ${ }^{51}$ that the performance of molecular junctions with large grains and grain boundaries is similar to that of junctions with small grains and grain boundaries

4 because the fraction of expose grain boundaries in the former is small ${ }^{36,52}$ ), following a wellestablished procedure (Section S2) and characterized in detail (Section S3). Figure 2 shows the angle-resolved X-ray photoelectron spectroscopy (AR XPS) results for the S $2 p$ signals for the

7 SAMs on the three different bottom electrodes recorded at normal $\left(90^{\circ}\right)$ and grazing $\left(40^{\circ}\right)$ take-

8 off angles (see Section S3 for the C 1s, Ag 3d, Au 4f, and Pt 4f spectra). The spectra are

9 dominated by two doublets each consisting of the S $2 p_{1 / 2}$ and S $2 p_{3 / 2}$ signals with the characteristic intensity ratio of 1:2. We assigned peak I with a binding energy of $\sim 162.0$ eV (blue

11 line) to the metal-thiolate bond and peak II with a binding energy of $\sim 163.9 \mathrm{eV}$ (red line) to the 5

12 sulfur atoms at the top of $\mathrm{S}\left(\mathrm{CH}_{2}\right)_{11} \mathrm{~S}-\mathrm{BTTF}$ SAMs and the physisorbed sulfur species, following previous peak assignments. ${ }^{53}$ For $\mathrm{S}\left(\mathrm{CH}_{2}\right)_{11} \mathrm{~S}-\mathrm{BTTF}$ SAMs on $\mathrm{Au}$, a small peak III (green line) at

$14 \quad 161.2 \mathrm{eV}$ is visible, which is attributed to Au-S bond associated with a disordered chemisorbed

15 phase. ${ }^{54-55}$ The XPS spectra recorded with $40^{\circ}$ take-off angle show that the intensity of peak I decreases with respect to peaks II (Figure 2), confirming that peak I originates from sulfur

17 species close to the metal surface (Au-S) and that peaks II originates from sulfur species located 18 at the top of the SAMs. 

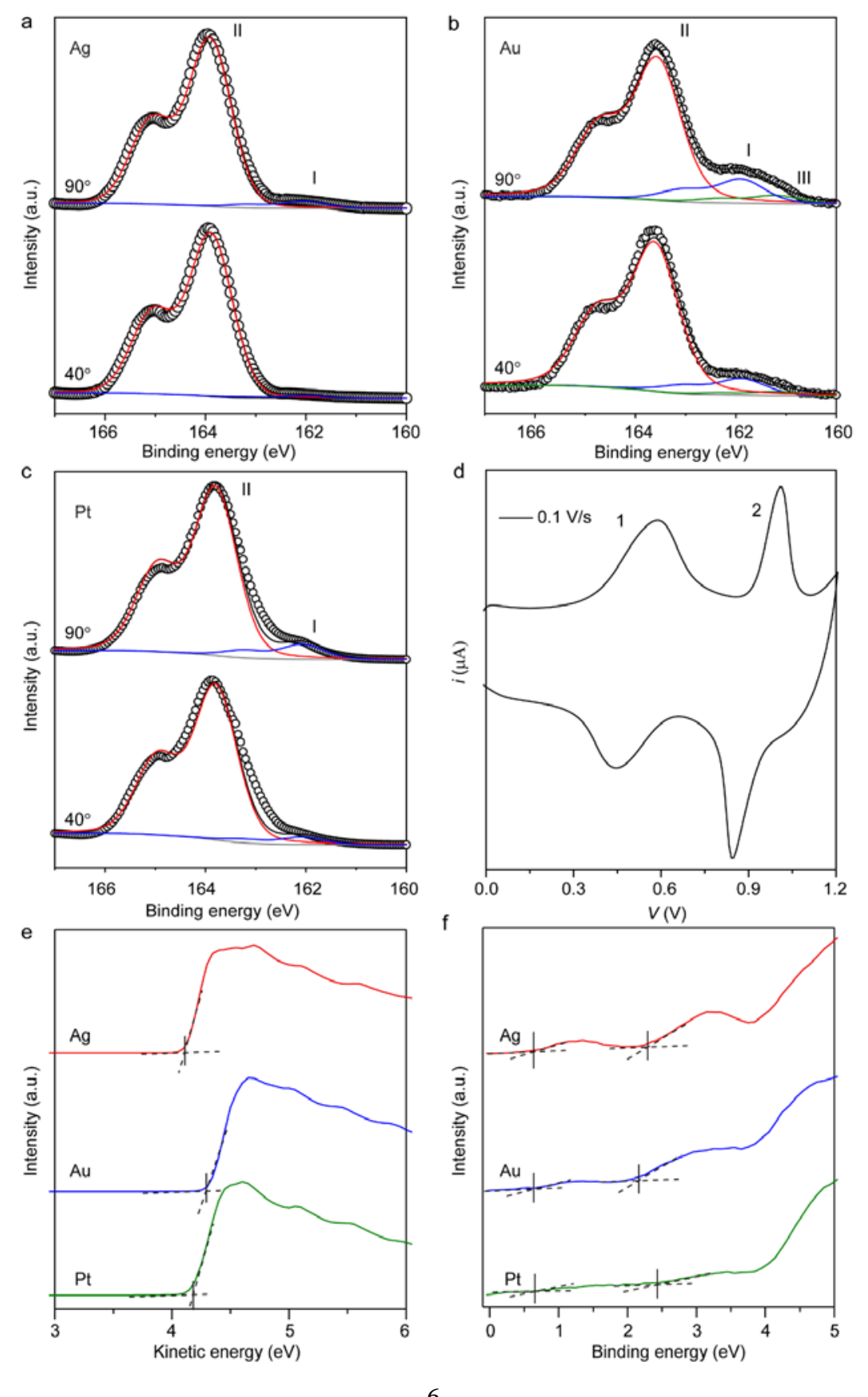
1 Figure 2. S $2 p$ spectra of $\mathrm{S}\left(\mathrm{CH}_{2}\right)_{11} \mathrm{~S}-\mathrm{BTTF}$ SAMs on $\mathrm{Ag}(\mathrm{a}), \mathrm{Au}(\mathrm{b})$, and Pt (c) at take-off angles of $90^{\circ}$ and $40^{\circ}$. (d) $\mathrm{CV}$ of S(CH$)_{11} \mathrm{~S}-\mathrm{BTTF}$ SAMs on Au at $0.1 \mathrm{~V} / \mathrm{s}$. (e) Secondary electron cutoff (SECO) spectra of S(CH$)_{11}$ S-BTTF SAMs on Ag, Au and Pt. (f) Valence band spectra of BTTF SAMs on Ag, Au and Pt (See Figure S6 for full valence band spectra). The HOMO and HOMO-1 onsets are marked with black lines.

From the AR XPS data, we determined the values of the SAM thickness $d_{\mathrm{SAM}}$ (Section S3) along the surface normal on Ag, Au, and Pt. The values of $d_{\mathrm{SAM}}$ are similar with experimental error (21 $\pm 3 \AA$ for Ag and Pt, $26.1 \pm 3 \AA$ for Au) and comparable to the molecular length (21.6 §) calculated using the CPK model and a tilt angle (relative to the surface normal) of $57^{\circ}$ determined by NEXAFS. We note that the somewhat larger value of $d_{\mathrm{SAM}}$ for $\mathrm{Au}$

12 originates from the disorder observed in the aforementioned XPS data. We found that the surface coverage $\left(\Gamma_{\mathrm{BTTF}}\right)$ of $\mathrm{S}\left(\mathrm{CH}_{2}\right)_{11} \mathrm{~S}-\mathrm{BTTF}$ SAMs relative to the values determined from $\mathrm{CV}$ (Section

$14 \mathrm{~S} 3$ ) are similar for all three metal surfaces (on average $\Gamma_{\mathrm{BTTF}}=1.88 \times 10^{-10} \mathrm{~mol} / \mathrm{cm}^{2}$; Table S1).

15 The similar tilt angle and surface coverage suggest that the SAM packing is driven by $\pi-\pi$ interactions between the BTTF units rather than differences in the M-S bond geometries, alkyl-

17 alkyl chain interactions or Gauche effects ${ }^{56-59}$.

Figure $2 \mathrm{~d}$ shows the cyclic voltammogram (CV) of the $\mathrm{S}\left(\mathrm{CH}_{2}\right)_{11} \mathrm{~S}-\mathrm{BTTF}$ SAMs on $\mathrm{Au}$ measured at a scan rate of $0.1 \mathrm{~V} / \mathrm{s}$. The CV exhibits two pairs of redox peaks with peak anodic/cathodic redox potentials $\left(E_{\mathrm{pa}} / E_{\mathrm{pc}}\right)$ of $+0.59 \mathrm{~V} /+0.44 \mathrm{~V}$ and $+1.01 \mathrm{~V} /+0.84 \mathrm{~V}$; these values are similar to those values determined with $\left(\mathrm{S}\left(\mathrm{CH}_{2}\right)_{11} \mathrm{~S}-\mathrm{BTTF}\right)_{2}$ in solution (Section S3). We assign peaks 1 and 2 to oxidation of BTTF unit to the radical cation and the dication, respectively, similar to SAMs with tetrathiafulvalene (TTF) termini ${ }^{60}$. The full width at half 
maximum (FWHM) of the second oxidation peak of the $\mathrm{S}\left(\mathrm{CH}_{2}\right)_{11} \mathrm{~S}-\mathrm{BTTF}$ SAMs on $\mathrm{Au}(88 \mathrm{mV})$ is smaller than that of the first oxidation peak $(167 \mathrm{mV})$. A similar behavior has also been observed in the CVs of other TTF SAMs on Au. ${ }^{60-61}$ For a reversible one-electron redox process, the FWHM is $90.6 \mathrm{mV}$ (at $25^{\circ} \mathrm{C}$ ) ${ }^{62}$, but here the broadening indicates strong repulsive electrostatic interactions between neighboring BTTF units, similar to previously reported SAMs with TTF termini. ${ }^{63}$ The system shows quasi-reversible behavior as the peak oxidation and reduction potentials of both peaks increase with increasing scan rates, which indicates sluggish heterogeneous electron transfer rates likely caused by the presence of the long alkyl chain

9 (Figure S9). Based on all these observations, we conclude that the SAMs are densely packed and 10 that the standing up phase dominates the SAM structure. Some of us have reported before that

11 SAMs derived from disulfides $\left(\left(\mathrm{S}-\left(\mathrm{CH}_{2}\right)_{11} \mathrm{Fc}\right)_{2}\right)$ are inferior to those derived from the

12 corresponding thiols ( $\left.\mathrm{HS}-\left(\mathrm{CH}_{2}\right)_{11} \mathrm{Fc}\right)$ with ferrocene $(\mathrm{Fc})$ head groups. ${ }^{54}$ In contrast, the BTTF

13 SAMs we report here readily form dense monolayers likely driven by favorable $\pi-\pi$ interactions

14 between neighboring BTTF units (which explains the similar tilt angles of the BTTF units of $57^{\circ}$ 15 as measured with NEXAFS).

16 Electronic structure of the SAMs. Figure $2 \mathrm{e}$ and $2 \mathrm{f}$ shows the secondary electron cutoff spectra 17 and the valence band, respectively (Section S3). For $\mathrm{S}\left(\mathrm{CH}_{2}\right)_{11} \mathrm{~S}-\mathrm{BTTF}$ SAMs on Ag, two features 18 just below the Fermi edge at a binding energy of 0.59 and $2.3 \mathrm{eV}$ are visible. Based on previous 19 peak assignments of ultraviolet photoelectron spectra (UPS) of TTF, ${ }^{64}$ we assign these two features to the HOMO and HOMO-1 from which we determined the energy of these levels with respect to vacuum, $E_{\mathrm{H}}$ and $E_{\mathrm{H}-1}$, respectively (Table 1). The intensities of the HOMO and HOMO-1 features for the SAMs on Au are smaller than those on Ag, and barely visible for

23 SAMs on Pt. As discussed in detail below, DFT calculations indicate that the different degree of 
hybridization between the molecular HOMO and HOMO-1 levels and the continuum of states of the metal electrode is responsible for this behavior (Section S5). We note that a similar decrease in the intensity of the HOMO and HOMO-1 features has been observed due to charge transfer

4 between TTF and tetracyanoquinodimethane (TCNQ) in TCNQ-TTF charge transfer crystals. ${ }^{64}$

5 We also determined the energy offset between HOMO and HOMO-1 and Fermi level of the

6 electrode, $\delta E_{\mathrm{H}}$ and $\delta E_{\mathrm{H}-1}$, which are similar for SAMs on Ag, Au and Pt (Table 1). These energy

7 offsets are comparable to what has been reported for TTF based SAMs on Au. ${ }^{53,65}$ From the

8 secondary electron cut-off spectra, we determined the work function $\Phi_{\text {SAM }}$ of the SAM-modified

9 bottom electrodes (Section S3). Table 1 shows that $\Phi_{\text {SAM }}$ for Ag and Pt surfaces are close to 4.1 $\mathrm{eV}$ while for Au surface, $\Phi_{\mathrm{SAM}}$ is $0.2 \mathrm{eV}$ larger which we attribute to the disorder of these SAMs,

11 as discussed earlier. Despite the large differences between the values of $\Phi_{\mathrm{M}}$ of the bare metal

12 ranging from $4.2 \mathrm{eV}$ for $\mathrm{Ag}$ to $5.75 \mathrm{eV}$ for Pt (Table 2), the values of $\Phi_{\mathrm{SAM}}$ are similar, which can be explained by Fermi level pinning, as discussed in more detail below. peak in near edge X-ray absorption fine structure (NEXAFS) spectra by following previously reported procedures (Section S3) and are listed in Table 1. The experimentally determined HOMO-LUMO gap from UPS and NEXAFS varies from 2.4 to $2.8 \mathrm{eV}$ (Table 1), and is comparable to the measured optical HOMO-LUMO gap of $2.99 \mathrm{eV}$ (Section S3) with UV/Vis spectroscopy. We note that the HOMO-LUMO gap measurements are rough estimates because of core-hole (in NEXAFS) and exciton (in UV/Vis) interactions, but qualitatively the different experimental values agree.

22 
1 Table 1. Summary of electronic properties of the $\mathrm{S}\left(\mathrm{CH}_{2}\right)_{11} \mathrm{~S}-\mathrm{BTTF}$ SAMs. More technical

2 details about the DFT (functional, k-point sampling, pseudopotentials, cut-offs) and unit cells

3 (lattice parameters, surface coverages) parameters used in our calculations are given in Section

$4 \quad$ S5 of the Supporting Information.

\begin{tabular}{cccccccc}
\hline \multicolumn{7}{c}{ UPS and NEXAFS } \\
\hline Metal & $\begin{array}{c}\Phi_{\mathrm{SAM}} \\
{[\mathrm{eV}]}\end{array}$ & $\begin{array}{c}\delta E_{\mathrm{H}} \\
{[\mathrm{eV}]}\end{array}$ & $\begin{array}{c}E_{\mathrm{H}} \\
{[\mathrm{eV}]}\end{array}$ & $\begin{array}{c}\delta E_{\mathrm{H}-1} \\
{[\mathrm{eV}]}\end{array}$ & $\begin{array}{c}E_{\mathrm{H}-1} \\
{[\mathrm{eV}]}\end{array}$ & $\begin{array}{c}\delta E_{\mathrm{L}} \\
{[\mathrm{eV}]}\end{array}$ & $\begin{array}{c}E_{\mathrm{L}} \\
{[\mathrm{eV}]}\end{array}$ \\
\hline $\mathrm{Ag}$ & 4.08 & 0.59 & -4.67 & 2.31 & -6.39 & 1.84 & -2.24 \\
$\mathrm{Au}$ & 4.31 & 0.60 & -4.91 & 2.15 & -6.46 & 2.25 & -2.06 \\
$\mathrm{Pt}$ & 4.16 & 0.62 & -4.78 & 2.42 & -6.58 & 1.83 & -2.33 \\
\hline
\end{tabular}

\begin{tabular}{ccccccc}
\hline \multicolumn{7}{c}{ DFT } \\
\hline$\Phi_{\mathrm{SAM}}$ & $\begin{array}{c}\delta E_{\mathrm{H}} \\
{[\mathrm{eV}]}\end{array}$ & $\begin{array}{c}E_{\mathrm{H}} \\
{[\mathrm{eV}]}\end{array}$ & $\begin{array}{c}\delta E_{\mathrm{H}-1} \\
{[\mathrm{eV}]}\end{array}$ & $\begin{array}{c}E_{\mathrm{H}-1} \\
{[\mathrm{eV}]}\end{array}$ & $\begin{array}{c}\delta E_{\mathrm{L}} \\
{[\mathrm{eV}]}\end{array}$ & $\begin{array}{c}E_{\mathrm{L}} \\
{[\mathrm{eV}]}\end{array}$ \\
\hline 4.01 & 0.12 & -4.12 & 1.47 & -5.48 & 2.16 & -1.85 \\
4.03 & 0.10 & -4.12 & 1.45 & -5.48 & 2.2 & -1.83 \\
3.99 & 0.11 & -4.10 & 1.49 & -5.48 & 2.25 & -1.74 \\
\hline
\end{tabular}

5

6 Electrical characterization of the Junctions. The junctions were fabricated by contacting the

$7 \quad$ SAMs with cone-shaped EGaIn tips (Figure 1) following a previously reported procedure ${ }^{36}$

8 (Section S4). The current density ( $J$ ) vs. voltage $(V)$ curves were recorded in the bias window of

$9 \pm 1.0 \mathrm{~V}$ for junctions on $\mathrm{Ag}, \pm 1.5 \mathrm{~V}$ for junctions on $\mathrm{Au}$, and $\pm 2.5 \mathrm{~V}$ for junctions on Pt; these

10 bias voltage ranges are below the breakdown voltages of EGaIn junctions with these bottom

11 electrodes. ${ }^{45}$ Table S3 summarizes the yields of working junctions, the number of junctions and

12 traces that were recorded and statistically analyzed following previously reported procedures to

13 obtain the Gaussian log-average $J(V)$ curves $\left(<\log _{10}|J|>_{G}(V)\right)$ along with the Gaussian log-

14 standard deviations and 95\% confidence levels shown in Figure 3. ${ }^{8}, 36,66$ Similarly, we also

15

16

17

18

19

20

21

constructed the histograms of $\log _{10}|R|$ at $\pm 1.0 \mathrm{~V}$ for $\mathrm{M}=\mathrm{Ag}, \pm 1.5 \mathrm{~V}$ for $\mathrm{M}=$ Au and $\pm 2.5 \mathrm{~V}$ for

$\mathrm{M}=$ Pt to determine $\left\langle\log _{10}|R|\right\rangle_{\mathrm{G}}$ and $\sigma_{\log }$ (Section S4).

The most striking result is that for junctions with $\mathrm{M}=\mathrm{Ag}$ no rectification is observed, while for junctions with $\mathrm{M}=\mathrm{Au}$ the value of $R^{+}$is $83\left(\sigma_{\log }=0.29\right)$ and $\mathrm{M}=\mathrm{Pt}$ the value of $R^{-}=9.1 \times$ $10^{2}\left(\sigma_{\log }=0.76\right)$. In other words, the direction of rectification is reversed for junctions with $\mathrm{M}=$ Au or Pt, implying that junctions with $\mathrm{M}=\mathrm{Au}$ allow the current to flow across the junction at positive bias while the opposite is true for junctions with $\mathrm{M}=$ Pt. The values of $J$ for the 
1 junctions with the Ag electrode are low, which indicates that within the bias window of $\pm 1.0 \mathrm{~V}$,

2 no molecular frontier orbitals enter the conduction window. In contrast, for junctions with Au

3 and Pt electrode, a large increase in the $J$ value is observed around $0.50 \mathrm{~V}$ and $0.90 \mathrm{~V}$,

4 respectively, which indicates that at these voltages, a molecular frontier orbital enters the

5 conduction window (see below). This increase in the $J$ value in only one bias polarity is reflected

6 in the corresponding $R(\mathrm{~V})$ plots (Figure 3). 

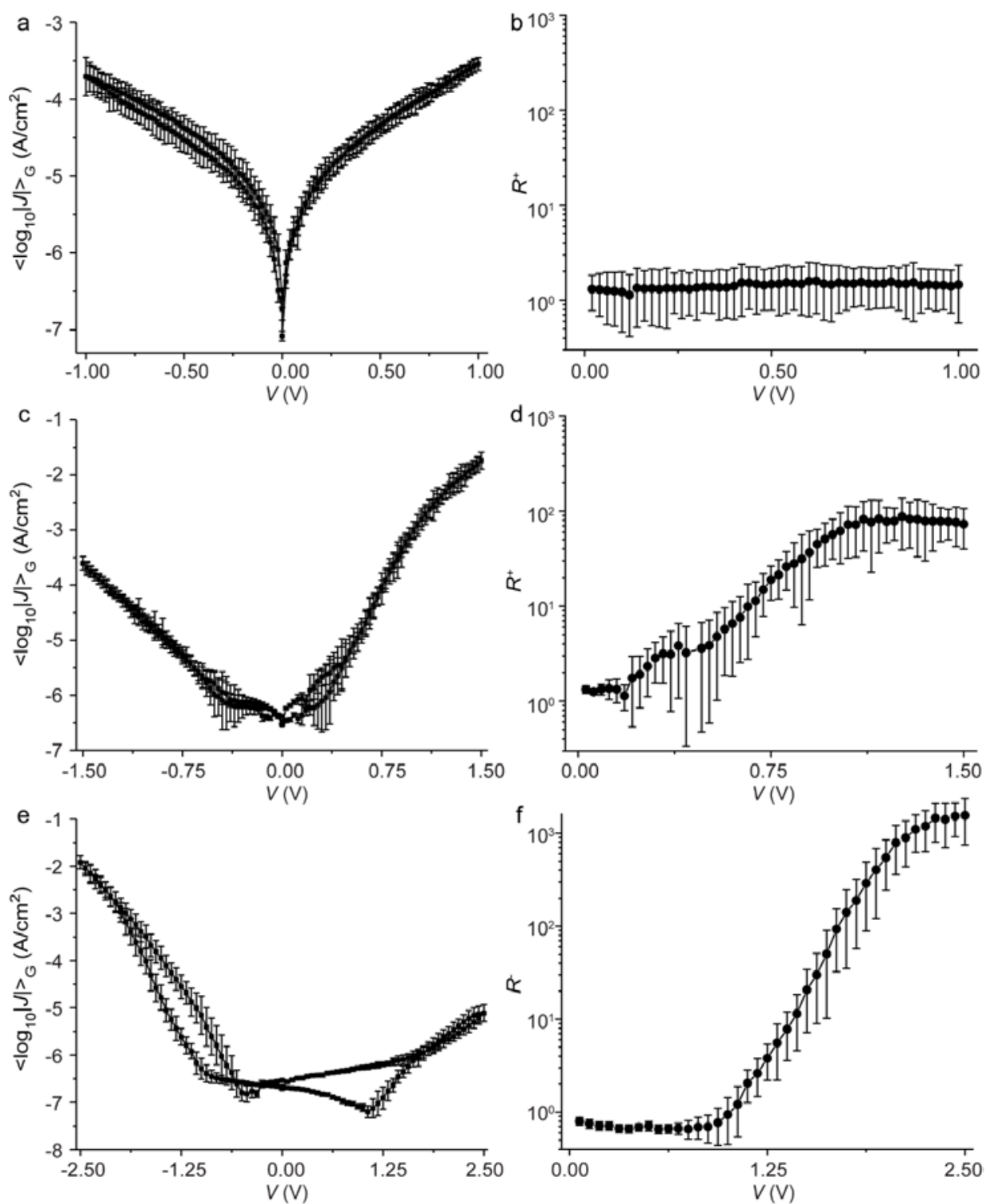

2 Figure 3. The plots of $\left\langle\log _{10}|J|>_{\mathrm{G}} \text { vs. } V \text { and } R(\mathrm{~V}) \text { plots obtained from M-S(CH} 2\right)_{11} \mathrm{~S}-$

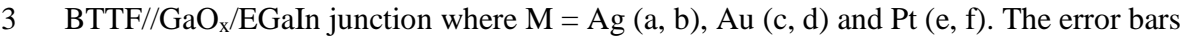



junctions (out of 29) for $M=A g, 26$ working junctions (out of 31) for $M=A u$ and 31 working

3 junctions (out of 39) for $\mathrm{M}=\mathrm{Pt}$.

5 Computational modeling of the self-assembled monolayers. We performed DFT calculations

6 to shed light on the electronic properties of the $\mathrm{S}\left(\mathrm{CH}_{2}\right)_{11} \mathrm{~S}$-BTTF based SAMs adsorbed on Ag,

7 Au and Pt surfaces (Section S5). The optimized structures display very similar geometric

8 parameters (Figures S12 and 13 and Tables S4 and S5) of the $\mathrm{S}\left(\mathrm{CH}_{2}\right)_{11} \mathrm{~S}-\mathrm{BTTF}$ molecules within

9 the SAM, as expected by the fact that the three metals are characterized by very similar lattice

10 parameters. This is in line with the experimental observations pointing to similar degree of

11 coverages, SAM thickness and tilt angles for the three studied SAMs. The only appreciable

12 difference is the metal-sulphur bond distance, which in the case of Pt, is about $0.2 \AA$ shorter with respect to Ag and Au surfaces as reported by others ${ }^{67}$. Due to the stronger Pt-S bonds, Pt$\mathrm{S}\left(\mathrm{CH}_{2}\right)_{11} \mathrm{~S}-\mathrm{BTTF}$ based SAMs are more stable (1.0 eV difference in the adsorption energy $\left.E_{\text {ads }}\right)$ compared with the other metal surfaces (Table S5).

17 three different surfaces). All $\Phi_{\text {SAM }}$ values are similar and close to $4.0 \mathrm{eV}$ due to the Fermi level

18 pinning, which is consistent with the $\Phi_{S A M}$ values determined with UPS ( 4.2 eV) despite the large differences in the work functions of the native metals ( $\Phi_{\mathrm{M}}$ in Table 2 ).

21 Table 2. Calculated electronic properties for the $\mathrm{S}\left(\mathrm{CH}_{2}\right)_{11} \mathrm{~S}-\mathrm{BTTF}$ SAMs adsorbed on Ag, $\mathrm{Au}$

22 and Pt metal surfaces.

\begin{tabular}{ccccccc}
\hline$M$ & $E_{\text {ads }}(\mathrm{eV})$ & $\Phi_{\mathrm{M}}(\mathrm{eV})$ & $\Phi_{\mathrm{SAM}}(\mathrm{eV})$ & $\Delta \Phi(\mathrm{eV})$ & $\Delta V_{\mathrm{SAM}}(\mathrm{eV})$ & $B D(\mathrm{eV})$ \\
\hline $\mathrm{Ag}$ & -2.32 & 4.2 & 4.01 & -0.19 & -1.65 & 1.46 \\
$\mathrm{Au}$ & -2.22 & 5.2 & 4.03 & -1.18 & -1.64 & 0.47 \\
$\mathrm{Pt}$ & -3.3 & 5.75 & 3.99 & -1.76 & -1.68 & -0.08 \\
\hline
\end{tabular}


2 down the total shift in two contributions originating from (Eq. 2): (i) the permanent dipole

3 ( $\left.\Delta V_{S A M}\right)$ of the molecular backbone (i.e., dipole mostly oriented along the BTTF unit) and (ii) the bond dipole contribution $(B D)$ which is the potential shift arising from the charge reorganization

5 upon adsorption.

$$
\Delta \Phi=\Delta V_{S A M}+B D
$$

$7 \quad(2)$

8 The values of $\Delta V_{S A M}$ are identical $(-1.65 \mathrm{eV})$ for the three studied surfaces due to the similar

9 geometric pattern of the $\mathrm{S}\left(\mathrm{CH}_{2}\right)_{11} \mathrm{~S}-\mathrm{BTTF}$ layers. For that reason, the origin of the pinning effect mentioned above is exclusively due to interfacial charge transfer upon adsorption. Variations in

$11 \mathrm{M}$-S dipole balancing the work function for different metals after SAM deposition has also

12 been observed for oligophenylene thiol SAMs both at experimental ${ }^{68}$ and theoretical ${ }^{69}$ levels.

13 Furthermore, this effect was also found in perylene diimide (PDI) SAMs with isocyanide

14 anchoring groups. ${ }^{70}$

We have analyzed the differences in charge density redistribution at the metal-molecule interfaces upon deposition of the SAM by following the so-called radical scenario (Eq. 3). In this formalism, the reorganization of the electron density at the interface ( $\Delta \rho$ in $\left.\mathrm{e}^{-} / \AA\right)$ can be estimated as the difference between the electron density of the full system ( $\left.\rho_{\mathrm{M}-\mathrm{SAM}}\right)$ and their individual components: the $\mathrm{S}\left(\mathrm{CH}_{2}\right)_{11} \mathrm{~S}-\mathrm{BTTF}$ layer $\left(\rho_{\mathrm{SAM}}\right)$ and the metal surface $\left(\rho_{\mathrm{M}}\right)$.

$$
\Delta \rho_{\rho}=\rho_{\mathrm{M}-\mathrm{SAM}}-\rho_{M}
$$

By cumulative integration of the electron density redistribution at the interface, we can estimate the charge transfer profile at the interface ( $\Delta Q$ in e-). Finally, the progressive buildup of the $B D$ 

obtained by applying the Poisson equation (Eq. 4) where $\varepsilon_{0}$ is the dielectric constant of vacuum.

$$
\nabla^{2} B D(z)=-\frac{\Delta \rho(z)}{\varepsilon_{0}}
$$

Figure 4 shows the different profiles of the charge transfer at the interface depending on

6 the metal surface. Interestingly, both the magnitude and direction of the charge transfer are changing as a function of the nature of metal surface: Ag and Au lose electron density, while Pt gains electron density. To obtain a quantitative estimation and a spatial representation of the

9 charge transfer mechanism, we performed a Hirshfeld versus Voronoi charge population 10 analysis $^{71}$ (Tables S6 and S7). The magnitude of the charge transfer is $-0.14,-0.02$, and $0.18 \mathrm{e}^{-}$

11 for $\mathrm{M}=\mathrm{Ag}$, Au and Pt, respectively. These values are consistent with reported charge transfers

12 found in other thiolate-based SAMs ${ }^{72-73}$. Despite the fact that $\mathrm{M}=\mathrm{Pt}$ presents higher values for 13 the charge transfer, the magnitude of the bond dipole associated with this transfer is very low.

14 Actually, the charge transfer for this surface is localized at the Pt-S interface, while in the case of 15 Ag and Au, a charge transfer between the BTTF unit and the metal surface is also found in addition to that associated with the $\mathrm{M}-\mathrm{S}$ bond (Figure S14). 


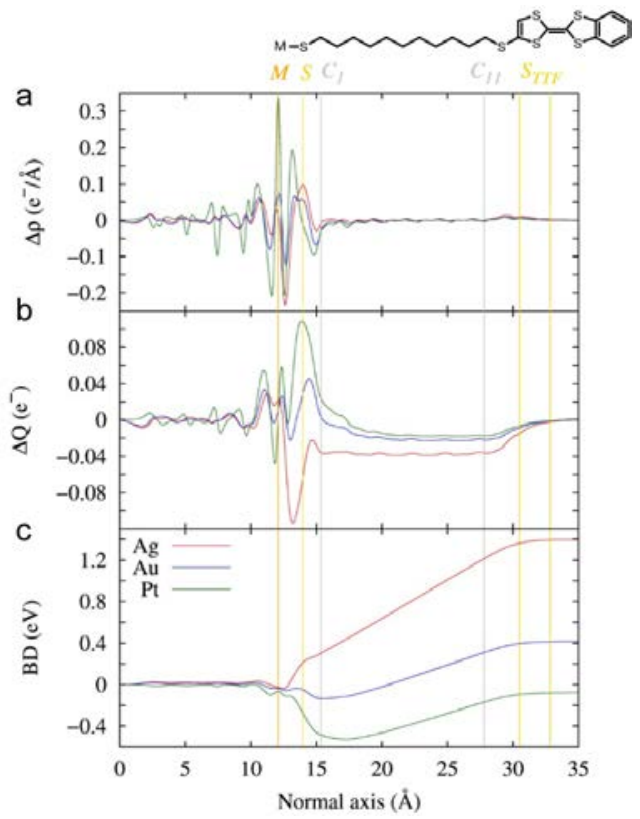

2 Figure 4. Plane averaged charge density difference (a), cumulated charge transfer (b) and bond

3 dipole (c) profile at the interface along the normal axis to Ag (red), Au (blue) and Pt (green)

4 metal surfaces. The vertical lines represent the atomic positions of the first metallic layer

5 (orange), anchoring group $\mathrm{S}$ (yellow), the first $\mathrm{C}$ bonded to the $\mathrm{S}\left(\mathrm{C}_{1}\right)$ and the $\mathrm{C}$ connected to the $6 \quad$ BTTF unit ( $\mathrm{C}_{11}$; grey).

7

Figure 5 shows the calculated energy level alignment of the M-S(CH$)_{11} \mathrm{~S}-\mathrm{BTTF}$ SAMs

on Ag, Au, and Pt. The combined M-S dipole and the partial charge transfer from the electrodes to BTTF units shifts the $E_{\text {HOMO }}$ centered at the BTTF unit toward $E_{\mathrm{f}}$, while the $E_{\mathrm{Lumo}}$ is shifted away from $E_{\mathrm{f}}$ for by an equal amount. The partial charge transfer to the BTTF unit is larger for

$12 \mathrm{M}=\mathrm{Ag}$, with respect to for $\mathrm{M}=\mathrm{Au}$, and $\mathrm{M}=\mathrm{Pt}$. Therefore, the shift in the potential associated 13 to the interfacial charge transfer (BD) is $1.46,0.47$ and $-0.08 \mathrm{eV}$ for $\mathrm{M}=\mathrm{Ag}$, $\mathrm{Au}$ and $\mathrm{Pt}$ 
respectively, which coincides with the above mentioned shift experimented by the energetic levels of the molecules. Consequently, the energy level alignment of the three interfaces is similar despite the large difference in work function of the native metals, in good agreement with

4 experiments (Table 1). We note that the mechanism of interfacial charge transfer between $\mathrm{M}$ and

5 BTTF most likely proceeds via a super exchange mechanism well-known for donor-bridge-

6 acceptor compounds ${ }^{48-50}$; here, the metal fulfils the role of the donor and BTTF that of the acceptor. This charge transfer does not reflect a formal reduction of the BTTF unit but actually corresponds to a fractional charge transfer.

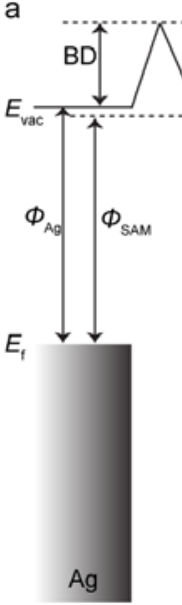

b
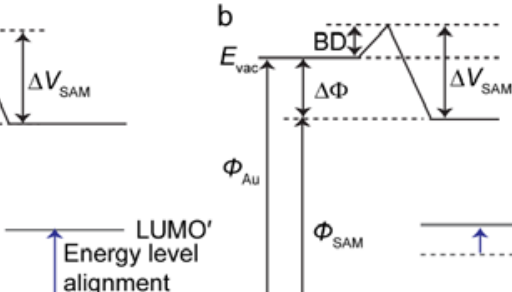
alignment

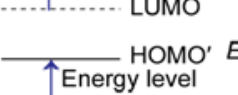
alignment
9

10

11

12

13

14

15

Figure 5. The computed energy level diagrams with $E_{\mathrm{vac}}=$ energy of vacuum, $E_{\mathrm{f}}=$ Fermi-level energy, $\Phi_{\mathrm{M}}=$ work function of metal $\left(\mathrm{M}=\mathrm{Ag}(\mathrm{a}), \mathrm{Au}(\mathrm{b})\right.$ and $\mathrm{Pt}(\mathrm{c}) . \Phi_{\mathrm{SAM}}=$ work function of the metal surface after SAM formation. HOMO and HOMO' are the highest occupied molecular orbitals before (black dashed lines) and after (black solid line) charge transfer with the Ag, Au and Pt bottom electrodes. LUMO (grey dashed lines) and LUMO' (grey solid line) are the corresponding lowest unoccupied molecular orbitals levels. 
In addition, the aforementioned pinning effect is at the origin of the similar energetic

3 alignment with respect to the metal $E_{\mathrm{f}}$ in the three studied SAMs (Figure 5), as it has been found

4 by UPS measurements for Ag and Au metal surfaces. Nevertheless, the HOMO signals are

5 barely visible in the UPS spectrum of Pt. The total density of states (DOS) of the different SAMs

6 shows that the states of Pt are very intense in the vicinity of the $E_{\mathrm{f}}$ (Figures S15 and 16).

7 Consequently, the hybridization of the $\mathrm{S}\left(\mathrm{CH}_{2}\right){ }_{11} \mathrm{~S}-\mathrm{BTTF}$ frontier occupied levels with the Pt

8 surface is higher than for the other electrodes (Figure S17). This finding agrees with the higher

9 amplitude of the oscillations in the evolution of $\Delta \rho$ with the normal distance with respect to that

10 across the Ag and Au metal surfaces (Figure 4a) and with the shorter $\mathrm{M}-\mathrm{S}$ bond distances. In

11 summary, stronger coupling implies a larger broadening of the molecular levels and hence a

12 lower resolution in the UPS spectra.

14 Computational modeling of the molecular junctions. To model the junction characteristics,

15 we placed a Ag top electrode on top of the $\mathrm{M}-\mathrm{S}\left(\mathrm{CH}_{2}\right)_{11} \mathrm{~S}-\mathrm{BTTF}$ SAMs with $\mathrm{M}=\mathrm{Ag}$, $\mathrm{Au}$, and $\mathrm{Pt}$

16 (Figures S18 and S23) (Section S5), because Ag has a similar work function ${ }^{74}\left(\Phi_{\mathrm{Ag}}=4.25 \mathrm{eV}\right)$ as

17 the $\mathrm{GaO}_{\mathrm{x}} \backslash \mathrm{EG}$ aIn top electrode ${ }^{75}\left(\Phi_{\mathrm{EGaIn}}=4.1-4.2 \mathrm{eV}\right)$. We also verified replacing Ga-In with Ag

18 top contacts has only a marginal effect on the transmission properties of the junctions (Figures

19 S23 and S24). However, our results are sensitive to the choice of van der Waals distance between

20 the SAM and the top contact (Figures S27-29). In our calculations, we used an optimized

21 distance of $2.5 \AA$ as discussed in Section S6 and computed the transmission spectra at the

22 equilibrium (0 V) for the 3 junctions (Figure 6 and Figures S19-22). We note that an electron

23 transfer takes place between the BTTF unit and the top electrode (Figure S30) which induces an 
interface dipole which downshifts the energetic levels of the SAMs. Consequently, the HOMO level in the junctions is not anymore in resonance with $E_{\mathrm{F}}$ as it was the case in the SAMs but $\delta E_{\mathrm{H}}$ increases to $\sim 0.9 \mathrm{eV}$ as determined from the transmission spectra in Figure 6. Similar shifts are also observed for the LUMO levels.

The transmission spectra across the junctions as a function of voltage were calculated by coupling the non-equilibrium Green's function theory (NEGF) to the DFT formalism using the Quantum ATK 2017.2 package (Figure 6). Due to the Fermi level pinning effects with the bottom electrode described in detail in the previous section, the transmission at negative/positive

9 bias polarities is governed by unoccupied/occupied levels as indicated by the purple and oranges lines in Figure 6. From the transmission spectra, the origin of the reversal of rectification despite

11 similarities in energy level alignment becomes clear, as shown in Figure 7. In the case of Ag

12 junctions, no energy levels fall in the relatively narrow bias window of $\pm 1.0 \mathrm{~V}$ (Figure $6 a$ ). For junctions with $\mathrm{Au}, \delta E_{\mathrm{H}}$ is smaller than $\delta E_{\mathrm{L}}$ (Figure 6b) in agreement with the XPS and NEXAFS

14 results (Table 1 ) indicating that the HOMO dominates the mechanism of charge transport at

15 positive bias resulting in rectification (Figure 7b). The participating of HOMO into the charge transport mechanism was further confirmed by the temperature dependent $J(\mathrm{~V})$ measurements reported in ref. 76 which demonstrated that at positive bias, the charge transport is thermally activated. In contrast, for junctions with Pt, at large applied bias, the LUMO dominates the mechanism of charge transport at negative bias. Although the HOMO is still involved with charge transport at positive bias, its contribution to the charge transport is very weak in comparison with the LUMO level because another unoccupied orbital (LUMO+1) is very close in energy to the LUMO (Figure 6c) and contribute significantly to charge transport at large negative bias (Figure 7c) so that both levels are participating in the transport for $-2.5 \mathrm{~V}$. Thus, the 
calculations are in full agreement with the experimental data and explain in detail why the direction of rectification changes as a function of $\mathrm{M}$. We would like to highlight that the calculations also show that the mechanism of charge transport would be similar for the three types of junctions in the hypothetical case that Ag and Au based junction could withstand the

5 same large applied bias as Pt.
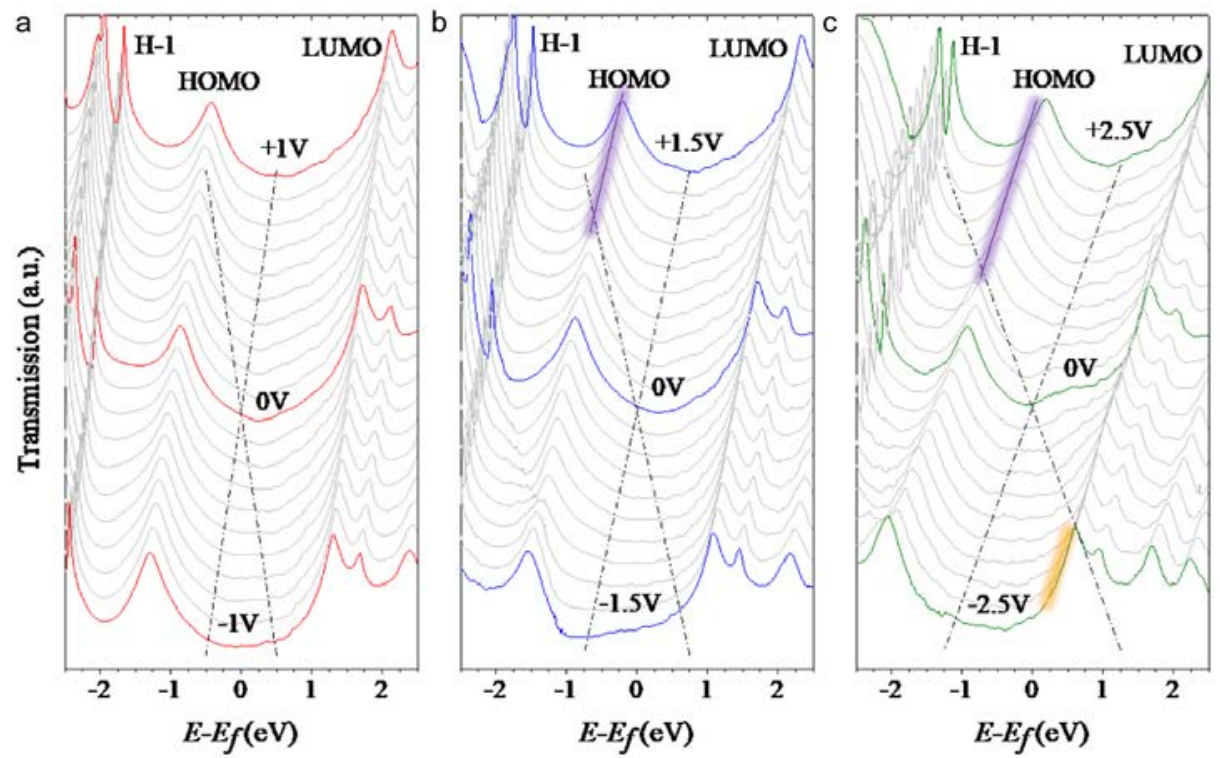

7 Figure 6. Transmission spectra across the $\mathrm{M}-\mathrm{S}\left(\mathrm{CH}_{2}\right)_{11} \mathrm{~S}-\mathrm{BTTF} / / \mathrm{Ag}$ junctions as a function of the voltage difference applied to the electrodes for $\mathrm{M}=\mathrm{Ag}$ (a), $\mathrm{Au}$ (b) and Pt (c). The cones

9 delimited by the dashed lines depict the voltage windows applied to each junction: $\pm 1 \mathrm{~V}$ for $\mathrm{M}=$ Ag, $\pm 1.5 \mathrm{~V}$ for $\mathrm{M}=\mathrm{Au}$ and $\pm 2.5 \mathrm{~V}$ for $\mathrm{M}=\mathrm{Pt}$. The purple and orange shades indicate when the HOMO or LUMO falls in the bias window, respectively. Note that the molecular orbital energies are not strictly coinciding with the energies of the transmission peaks. Nevertheless, no big differences in these energies are expected in view of the weak coupling between the BTTF

14 moiety and both electrodes. 
a

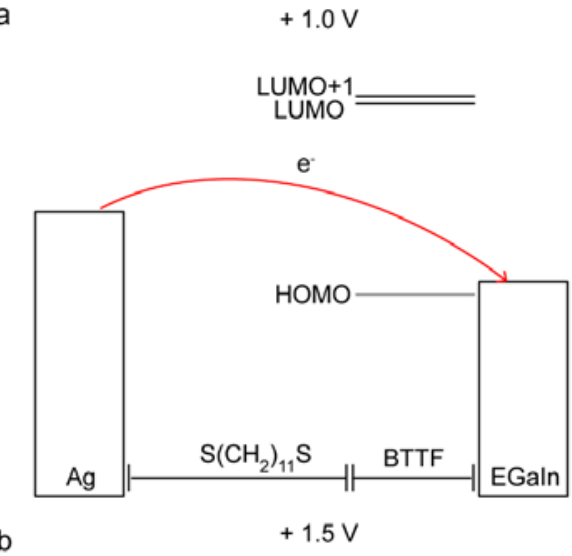

b

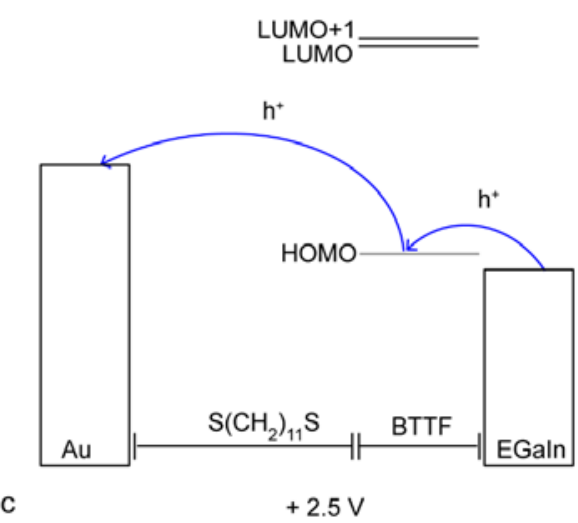

c

1

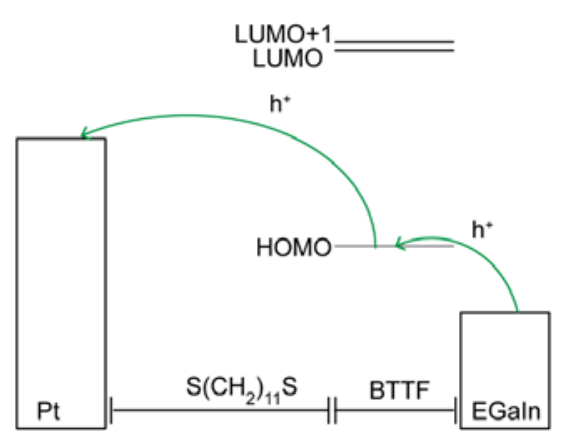

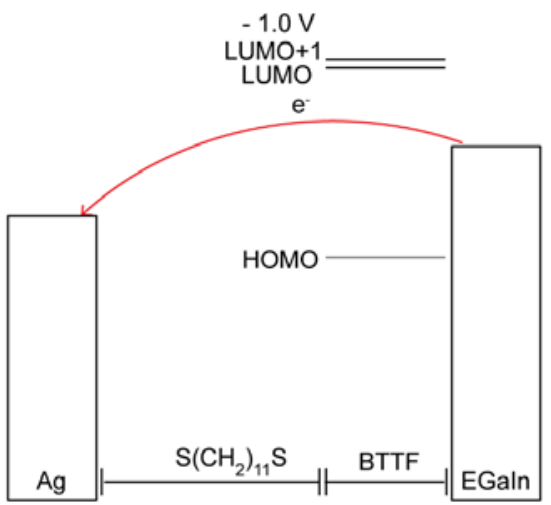

$-1.5 \mathrm{~V}$

LUMO+1 LUMO
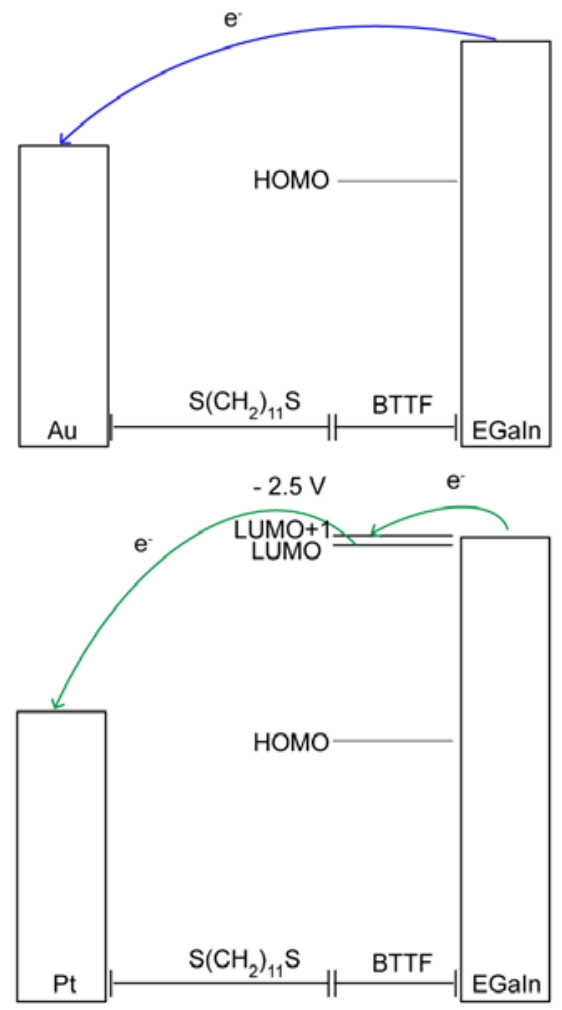
1 Figure 7. Energy level diagrams of $\mathrm{M}-\mathrm{S}\left(\mathrm{CH}_{2}\right)_{11} \mathrm{~S}-\mathrm{BTTF} / / \mathrm{GaO}_{\mathrm{x}} / \mathrm{EGaIn}$ junctions with $\mathrm{M}=\mathrm{Ag}$ (a),

$2 \mathrm{Au}(\mathrm{b})$ and Pt (c). $\Phi_{\mathrm{SAM}}$ and $\Phi_{\mathrm{EGaIn}}$ are the work function of the bottom electrode and top

3 electrodes after SAM formation. The arrows indicate the charge transport process. The energy

4 level diagrams were constructed based on the experimentally obtained $E_{\text {HOMO, }} E_{\text {LUMO }}$ and $\Phi_{\mathrm{SAM}}$

5 values from UPS and NEXAFS. A renormalization factor of 1.5 was used to correct for the

6 change of the energy offset when adding a second electrode. ${ }^{77}$

7

According to the UPS measurements, $\delta E_{\mathrm{H}} \approx 0.60 \mathrm{eV}$ (Table 1) but the calculations

9 suggest that this level should enter the bias window around 1V bias. Similarly, the NEXAFS results indicate that $\delta E_{\mathrm{H}} \approx 1.8 \mathrm{eV}$ while the calculations indicate that the LUMO enters the bias

11 window around $1.5 \mathrm{eV}$. However, in these measurements the top electrode is not present but the

12 DFT calculations show that charge transfer between the BTTF and top electrode results in a downshift of the energy levels of $0.5 \mathrm{eV}$, when moving from large to close top electrode contacts

14 (see Figure S27). We have estimated this downshift from the transition voltages both

15 theoretically (Section S5) and experimentally (Section S6) as the maximum of the $\mathrm{d} J / \mathrm{d} V \mathrm{vs}$. applied bias functions to identify the energetic positions of the conduction orbitals. We

17 performed normalized differential conduction (NDC) analysis using Eq. 5 for the individual $J(V)$ 18 traces. $^{78-80}$

$$
N D C=\frac{d J}{d V} \cdot \frac{V}{J}=\frac{d(\log J)}{d(\log V)}
$$

20 Figure 8a shows the NDC curve obtained from an Au-S( $\left.\mathrm{CH}_{2}\right)_{11} \mathrm{~S}-\mathrm{BTTF} / / \mathrm{GaO}_{\mathrm{x}} / \mathrm{EGaIn}$ junction

21 with a resonance peak at $+1.03 \mathrm{~V}$. This peak indicates that the HOMO enters the conduction

22 window at this bias. ${ }^{81-82}$ At negative bias, the NDC curve shows typical parabolic behavior with

23 NDC increasing to NDC $=3$ which is characteristic for off-resonant tunneling ${ }^{78}$. The NDC curve 
for a Pt-S( $\left.\mathrm{CH}_{2}\right)_{11} \mathrm{~S}-\mathrm{BTTF} / / \mathrm{GaO}_{\mathrm{x}} / \mathrm{EGaIn}$ junction (Figure $8 \mathrm{~b}$ ) is dominated by a resonance peak at

2 -1.79 $\mathrm{V}$ indicating that the LUMO dominates the mechanism of charge transport at this voltage.

3 Figure 8c-d shows the theoretical $\mathrm{d} J / \mathrm{d} V$ vs. $V$ curves which mimic the experimental NDC plots

4 very well. Figure $8 \mathrm{c}$ shows the HOMO resonance at $+0.9 \mathrm{~V}$ for junctions with $\mathrm{M}=\mathrm{Au}$ which

5 corresponds very well to the peak observed in the NDC plot shown in Figure 8a. Likewise,

6 Figure 8d shows a peak at $-2.0 \mathrm{~V}$ corresponding to a resonance of the LUMO which matches

$7 \quad$ very well the NDC plot shown in Figure. $8 \mathrm{~b}$ for the same junction. We note that the small

8 HOMO peak in Figure $8 \mathrm{~d}$ is visible at $1.0 \mathrm{~V}$, but this peak is not resolved in the experimental

9 data (Figure 8b). In the experimental data, the resonance for Pt is very broad. Likely at positive

10 bias the HOMO is too broad to be seen as a clear peak which could explain why the NDC curve

11 for Pt at positive bias does not follow a clear parabolic behavior but instead the NDC value

12 gradually increases with $V$. In summary, the agreement between experiments and theory indicates that introduction of the top-electrode results in a downshift in energies of the molecular

14 levels of about $0.4 \mathrm{eV}$.

However, the calculated increase of the currents once a molecular frontier orbital enters the conduction window are much lower than the experimentally observed ones. Therefore, the experimental rectification ratios are between one or two orders of magnitude higher that the calculated ratios (Figure S32). It is important to note that our theoretical approach is used to model the charge transport only in the coherent regime and not in the incoherent hopping regime. ${ }^{83-84}$ Preliminary data indicate that indeed hopping is important when a molecular frontier

21 orbital falls in the conduction window (similar to molecular diodes based on ferrocene units) ${ }^{36}$, but this change in the mechanism of charge transport from coherent to incoherent tunneling has not been accounted for in the current theory and therefore the theory might underestimate the 

reliable trends to rationalize experiments rather than a quantitative description of the energy level

3 alignment in the junction due to the inherent limitations of DFT to predict accurate electronic

4 gaps, especially in the proximity of metallic electrodes.
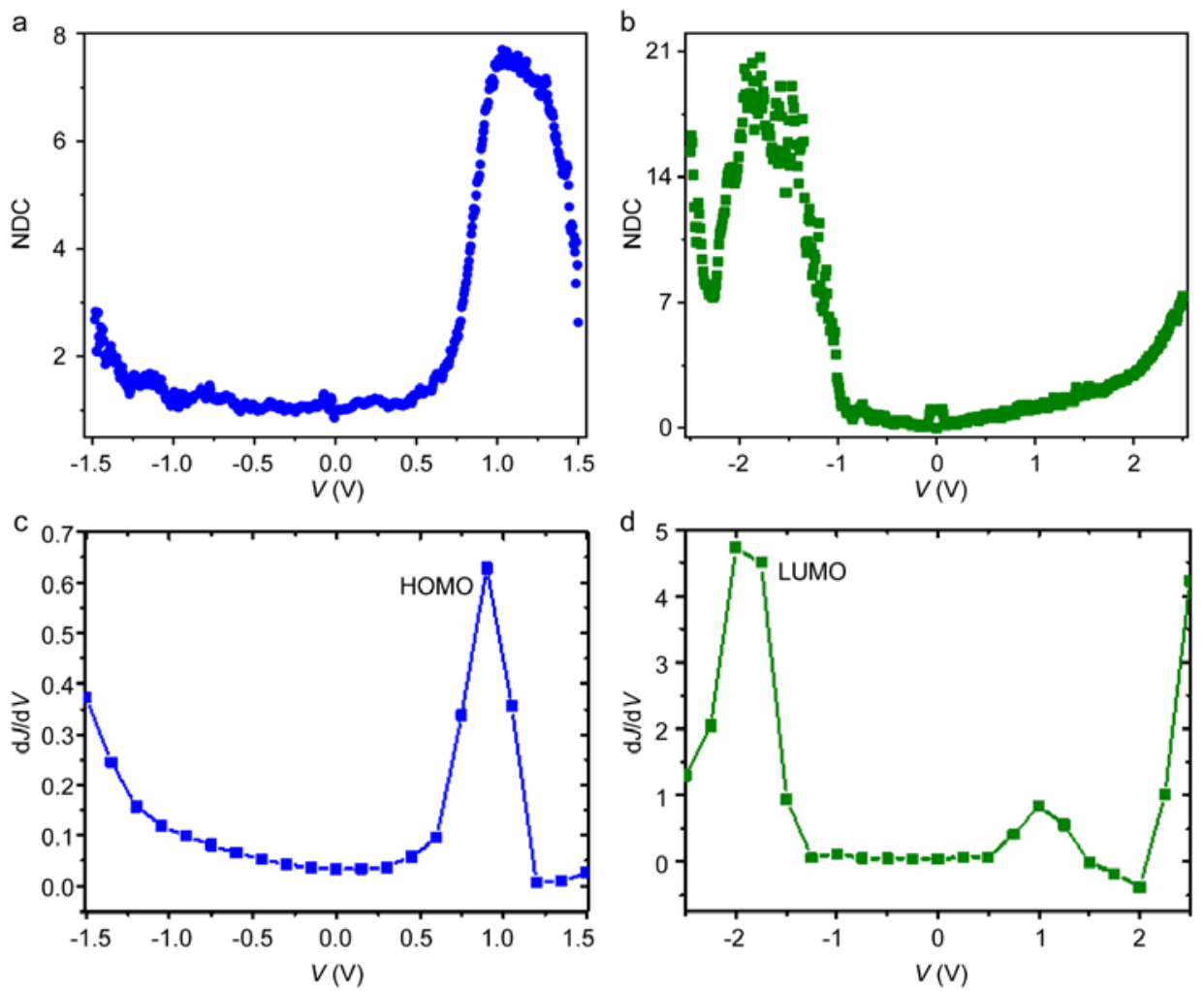

Figure 8. NDC plots for $\mathrm{M}-\mathrm{S}\left(\mathrm{CH}_{2}\right)_{11} \mathrm{~S}-\mathrm{BTTF} / / \mathrm{GaO}_{\mathrm{x}} / \mathrm{EGaIn}$ junction where $\mathrm{M}=\mathrm{Au}(\mathrm{a})$ and $\mathrm{Pt}(\mathrm{b})$.

7 Calculated $\mathrm{d} J / \mathrm{d} V$ vs. applied voltage curves for $\mathrm{Au}(\mathrm{c})$ and $\mathrm{Pt}(\mathrm{d})$ junctions.

8

9 


\section{Conclusions}

Usually it is challenging to tune the energy level alignment of molecular junctions due to

3 Fermi level pinning. Consequently, it is still not straightforward to control electronic functions of

4 junctions, such as the direction of rectification. Most approaches to control rectification focus on

5 chemical alteration of the molecular component of the junctions, but systematic studies involving

6 different electrode materials are rare. Changes in work function of the electrode have an effect on

7 the magnitude and/or direction of interface dipoles, but often this effect is masked when the

8 functional group couples too strongly with the electrode ${ }^{36-37}$. In this work, the BTTF redox unit is

9 decoupled from the bottom electrode by the alkyl chain making it possible to investigate interfacial charge transfer across the metal-thiolate bond and from the metal to the BTTF unit in

11 detail. With the aid of detailed DFT calculations, the contributions of charge transfer across the

12 metal-molecule interface involving the thiol-anchoring group and the redox-active unit in the

13 SAMs as a function of three different metals with large differences in work functions $(\mathrm{M}=\mathrm{Ag}$,

$14 \mathrm{Au}$, and Pt) were elucidated. By using different metals, the bias window could be increased from $15 \pm 1.0 \mathrm{~V}$ for Ag to $\pm 2.5 \mathrm{~V}$ for Pt making it possible to change the mechanism of rectification from HOMO dominated charge transport at low applied bias for junctions with Au to LUMO

17 dominated charge transport for junctions with Pt at large bias. The change in the energy level alignment of the system upon the application of a certain bias resulted in reversal of rectification

19 in molecular diodes with EGaIn top electrodes. In other words, we have demonstrated a new way 20 of tuning the direction of rectification by changing the bottom electrode material of the junction rather than by chemical alteration of the molecular structure which involves (often timeconsuming) chemical synthesis. Our work provides new insights in the understanding of energy 
level alignment in molecular junctions with redox groups which provide energetically accessible energy levels and is important toward the rational design of molecular junctions in general.

\section{ASSOCIATED CONTENT}

\section{Supporting Information}

6 The Supporting Information is available free of charge on the ACS Publications website.

7 Synthesis of $\left(\mathrm{S}\left(\mathrm{CH}_{2}\right)_{11} \mathrm{~S}-\mathrm{BTTF}\right)_{2}$, preparation of self-assembled monolayers, characterization of

8 the self-assembled monolayers, junction fabrication and statistical analysis, DFT calculations.

10 AUTHOR INFORMATION

\section{Corresponding Authors}

12 Jerome Cornil-Laboratory for Chemistry of Novel Materials, University of Mons, Place du Parc

13 20, B-7000, Mons, Belgium. ORCID: https://orcid.org/0000-0002-5479-4227. Email:

14 jerome.cornil@umons.ac.be

15 Jaume Veciana- Institut de Ciència de Materials de Barcelona (ICMAB-CSIC)/CIBER-BBN,

16 Campus de la UAB, 08193 Bellaterra, Spain. ORCID: https://orcid.org/0000-0003-1023-9923.

17 Email: vecianaj@icmab.es

18 Christian A. Nijhuis- Department of Chemistry and Centre for Advanced 2D Materials,

19 National University of Singapore, Singapore 117543 Singapore. ORCID: https://orcid.org/0000-

20 0003-3435-4600. Email: chmnca@nus.edu.sg

21

22 Authors

23 Yingmei Han- Department of Chemistry, National University of Singapore, 3 Science Drive 3,

24 Singapore 117543, Singapore 
Maria Serena Maglione- Institut de Ciència de Materials de Barcelona (ICMAB-CSIC)/CIBER-

BBN, Campus de la UAB, 08193 Bellaterra, Spain

3 Valentin Diez Cabanes- Laboratory for Chemistry of Novel Materials, University of Mons,

4 Place du Parc 20, B-7000, Mons, Belgium. https://orcid.org/0000-0002-6234-2749

5 Javier Casado-Montenegro- Institut de Ciència de Materials de Barcelona (ICMAB-

6 CSIC)/CIBER-BBN, Campus de la UAB, 08193 Bellaterra, Spain

7 Xiaojiang Yu- Singapore Synchrotron Light Source, National University of Singapore, 5

8 Research Link, Singapore 117603 Singapore

9 Senthil Kumar Karuppannan- Department of Chemistry, National University of Singapore, 3

10 Science Drive 3, Singapore 117543, Singapore. ORCID: https://orcid.org/0000-0002-5956-0219.

11 Ziyu Zhang- Department of Chemistry, National University of Singapore, 3 Science Drive 3,

12 Singapore 117543, Singapore

13 Núria Crivillers- Institut de Ciència de Materials de Barcelona (ICMAB-CSIC)/CIBER-BBN,

14 Campus de la UAB, 08193 Bellaterra, Spain. ORCID: https://orcid.org/0000-0001-6538-2482

15 Marta Mas-Torrent- Institut de Ciència de Materials de Barcelona (ICMAB-CSIC)/CIBERBBN, Campus de la UAB, 08193 Bellaterra, Spain. ORCID: https://orcid.org/0000-0002-1586-

$17005 X$

18 Concepció Rovira- Institut de Ciència de Materials de Barcelona (ICMAB-CSIC)/CIBER-BBN,

\section{Notes}

22 The authors declare no competing financial interest.

23 


\section{ACKNOWLEDGEMENTS}

2 The authors express thanks to the Ministry of Education (MOE) for supporting this research 3 under Award No. MOE2018-T2-1-088 and R-143-000-B30-112. We also acknowledge the

4 Prime Minister's Office, Singapore, under its Medium Sized Centre program for supporting this

5 research. This work was also funded by ITN iSwitch 642196, the DGI (Spain), projects FANCY

6 CTQ2016-80030-R and MOTHER (MAT2016-80826-R), the Generalitat de Catalunya (2017-

7 SGR-918), the Instituto de Salud Carlos III, through “Acciones CIBER”, and the Spanish

8 Ministry of Economy and Competitiveness, through the "Severo Ochoa” program for Centers of

9 Excellence in R\&D (SEV-2015-0496). The work in Mons was financially supported by the EC

10 through the Marie Curie project ITN iSwitch (GA no. 642196). Computational resources were

11 provided by the Consortium des Équipements de Calcul Intensif (CÉCI) funded by the Belgian

12 National Fund for Scientific Research (F.R.S.-FNRS) under Grant 2.5020.11. J.C. is an FNRS

13 research director.

14

\section{REFERENCES}

16 1. Kaneko, M.; Okada, T., Charge Transport in Molecular Catalysis in a Heterogeneous Phase.

17 In Molecular Catalysts for Energy Conversion, Okada, T.; Kaneko, M., Eds. Springer Berlin

18 Heidelberg: Berlin, Heidelberg, 2009; pp 37-65.

19 2. Amdursky, N.; Marchak, D.; Sepunaru, L.; Pecht, I.; Sheves, M.; Cahen, D., Electronic

20 Transport Via Proteins. Advanced Materials 2014, 26, 7142-7161.

21 3. Wang, Q.; Ma, D., Management of Charges and Excitons for High-Performance White

22 Organic Light-Emitting Diodes. Chemical Society Reviews 2010, 39, 2387-2398. 
1 4. Du, W.; Han, Y.; Hu, H.; Chu, H.-S.; Annadata, H. V.; Wang, T.; Tomczak, N.; Nijhuis, C.

2 A., Directional Excitation of Surface Plasmon Polaritons Via Molecular through-Bond Tunneling

3 across Double-Barrier Tunnel Junctions. Nano Letters 2019, 19, 4634-4640.

$4 \quad$ 5. de Nijs, B.; Benz, F.; Barrow, S. J.; Sigle, D. O.; Chikkaraddy, R.; Palma, A.; Carnegie, C.;

5 Kamp, M.; Sundararaman, R.; Narang, P.; Scherman, O. A.; Baumberg, J. J., Plasmonic Tunnel

6 Junctions for Single-Molecule Redox Chemistry. Nature Communications 2017, 8, 994.

7 6. Adnan, M.; Baumberg, J. J.; Vijaya Prakash, G., Linear and Nonlinear Optical Probing of

8 Various Excitons in 2d Inorganic-Organic Hybrid Structures. Scientific Reports 2020, 10, 2615.

9 7. Urbieta, M.; Barbry, M.; Zhang, Y.; Koval, P.; Sánchez-Portal, D.; Zabala, N.; Aizpurua, J.,

10 Atomic-Scale Lightning Rod Effect in Plasmonic Picocavities: A Classical View to a Quantum

11 Effect. ACS Nano 2018, 12, 585-595.

12 8. Han, Y.; Nickle, C.; Zhang, Z.; Astier, H. P. A. G.; Duffin, T. J.; Qi, D.; Wang, Z.; del

13 Barco, E.; Thompson, D.; Nijhuis, C. A., Electric-Field-Driven Dual-Functional Molecular

14 Switches in Tunnel Junctions. Nature Materials 2020, 19, 843-848.

15 9. Kumar, S.; Merelli, M.; Danowski, W.; Rudolf, P.; Feringa, B. L.; Chiechi, R. C., Chemical

Locking in Molecular Tunneling Junctions Enables Nonvolatile Memory with Large on-Off

\section{Ratios. Advanced Materials 2019, 31, 1807831.}

10. Wani, I. H.; Jafri, S. H. M.; Warna, J.; Hayat, A.; Li, H.; Shukla, V. A.; Orthaber, A.;

Grigoriev, A.; Ahuja, R.; Leifer, K., A Sub 20 Nm Metal-Conjugated Molecule Junction Acting as a Nitrogen Dioxide Sensor. Nanoscale 2019, 11, 6571-6575.

11. Yan, H.; Bergren, A. J.; McCreery, R.; Della Rocca, M. L.; Martin, P.; Lafarge, P.; Lacroix, J. C., Activationless Charge Transport across 4.5 to $22 \mathrm{Nm}$ in Molecular Electronic Junctions.

Proceedings of the National Academy of Sciences 2013, 110, 5326. 

across Ferritin-Based Junctions. Advanced Materials 2016, 28, 1824-1830.

13. Yuan, L.; Wang, L.; Garrigues, A. R.; Jiang, L.; Annadata, H. V.; Anguera Antonana, M.; Barco, E.; Nijhuis, C. A., Transition from Direct to Inverted Charge Transport Marcus Regions in Molecular Junctions Via Molecular Orbital Gating. Nature Nanotechnology 2018, 13, 322-

6329.

14. Migliore, A.; Schiff, P.; Nitzan, A., On the Relationship between Molecular State and Single Electron Pictures in Simple Electrochemical Junctions. Physical Chemistry Chemical Physics 2012, 14, 13746-13753.

10 15. Atxabal, A.; Arnold, T.; Parui, S.; Hutsch, S.; Zuccatti, E.; Llopis, R.; Cinchetti, M.;

11 Casanova, F.; Ortmann, F.; Hueso, L. E., Tuning the Charge Flow between Marcus Regimes in 12 an Organic Thin-Film Device. Nature Communications 2019, 10, 2089.

13 16. Thomas, J. O.; Limburg, B.; Sowa, J. K.; Willick, K.; Baugh, J.; Briggs, G. A. D.; Gauger,

14 E. M.; Anderson, H. L.; Mol, J. A., Understanding Resonant Charge Transport through Weakly

15 Coupled Single-Molecule Junctions. Nature Communications 2019, 10, 4628.

16 17. Galperin, M.; Ratner, M. A.; Nitzan, A.; Troisi, A., Nuclear Coupling and Polarization in

17 Molecular Transport Junctions: Beyond Tunneling to Function. Science 2008, 319, 1056.

18 18. Taherinia, D.; Smith, C. E.; Ghosh, S.; Odoh, S. O.; Balhorn, L.; Gagliardi, L.; Cramer, C.

19 J.; Frisbie, C. D., Charge Transport in 4 Nm Molecular Wires with Interrupted Conjugation:

20 Combined Experimental and Computational Evidence for Thermally Assisted Polaron

21 Tunneling. ACS Nano 2016, 10, 4372-4383. 
19. Asadi, K.; Kronemeijer, A. J.; Cramer, T.; Jan Anton Koster, L.; Blom, P. W. M.; de Leeuw,

2 D. M., Polaron Hopping Mediated by Nuclear Tunnelling in Semiconducting Polymers at High

3 Carrier Density. Nature Communications 2013, 4, 1710.

4 20. Heimel, G.; Romaner, L.; Zojer, E.; Bredas, J.-L., The Interface Energetics of Self-

5 Assembled Monolayers on Metals. Accounts of Chemical Research 2008, 41, 721-729.

6 21. Braun, S.; Salaneck, W. R.; Fahlman, M., Energy-Level Alignment at Organic/Metal and

7 Organic/Organic Interfaces. Advanced Materials 2009, 21, 1450-1472.

8 22. Vilan, A.; Aswal, D.; Cahen, D., Large-Area, Ensemble Molecular Electronics: Motivation

9 and Challenges. Chemical Reviews 2017, 117, 4248-4286.

10 23. Cahen, D.; Kahn, A.; Umbach, E., Energetics of Molecular Interfaces. Materials Today

$112005,8,32-41$.

12 24. Amsalem, P.; Niederhausen, J.; Wilke, A.; Heimel, G.; Schlesinger, R.; Winkler, S.;

13 Vollmer, A.; Rabe, J. P.; Koch, N., Role of Charge Transfer, Dipole-Dipole Interactions, and

14 Electrostatics in Fermi-Level Pinning at a Molecular Heterojunction on a Metal Surface.

15 Physical Review B 2013, 87, 035440.

16 25. Hofmann, O. T.; Egger, D. A.; Zojer, E., Work-Function Modification Beyond Pinning:

17 When Do Molecular Dipoles Count? Nano Letters 2010, 10, 4369-4374.

18 26. Egger, D. A.; Zojer, E., Anticorrelation between the Evolution of Molecular Dipole

19 Moments and Induced Work Function Modifications. The Journal of Physical Chemistry Letters

$20 \quad 2013,4,3521-3526$.

21 27. Obersteiner, V.; Egger, D. A.; Heimel, G.; Zojer, E., Impact of Collective Electrostatic

22 Effects on Charge Transport through Molecular Monolayers. The Journal of Physical Chemistry

23 C 2014, 118, 22395-22401. 
28. Chen, X.; Annadata, H. V.; Kretz, B.; Zharnikov, M.; Chi, X.; Yu, X.; Egger, D. A.; Nijhuis,

2 C. A., Interplay of Collective Electrostatic Effects and Level Alignment Dictates the Tunneling

3 Rates across Halogenated Aromatic Monolayer Junctions. The Journal of Physical Chemistry

$4 \quad$ Letters 2019, 10, 4142-4147.

5 29. Aviram, A.; Ratner, M. A., Chem. Phys. Lett. 1974, 29, 277.

6 30. Bayat, A.; Lacroix, J.-C.; McCreery, R. L., Control of Electronic Symmetry and

7 Rectification through Energy Level Variations in Bilayer Molecular Junctions. Journal of the

8 American Chemical Society 2016, 138, 12287-12296.

9 31. Luo, L.; Balhorn, L.; Vlaisavljevich, B.; Ma, D.; Gagliardi, L.; Frisbie, C. D., Hopping

10 Transport and Rectifying Behavior in Long Donor-Acceptor Molecular Wires. The Journal of

11 Physical Chemistry C 2014, 118, 26485-26497.

12 32. Baghbanzadeh, M.; Belding, L.; Yuan, L.; Park, J.; Al-Sayah, M. H.; Bowers, C. M.;

13 Whitesides, G. M., Dipole-Induced Rectification across Agts/Sam//Ga2o3/Egain Junctions.

14 Journal of the American Chemical Society 2019, 141, 8969-8980.

15 33. Kovalchuk, A.; Egger, D. A.; Abu-Husein, T.; Zojer, E.; Terfort, A.; Chiechi, R. C., DipoleInduced Asymmetric Conduction in Tunneling Junctions Comprising Self-Assembled

17 Monolayers. RSC Advances 2016, 6, 69479-69483.

18 34. Ai, Y.; Kovalchuk, A.; Qiu, X.; Zhang, Y.; Kumar, S.; Wang, X.; Kühnel, M.; Nørgaard, K.;

19 Chiechi, R. C., In-Place Modulation of Rectification in Tunneling Junctions Comprising Self-

20 Assembled Monolayers. Nano Letters 2018, 18, 7552-7559.

21 35. Atesci, H.; Kaliginedi, V.; Celis Gil, J. A.; Ozawa, H.; Thijssen, J. M.; Broekmann, P.;

22 Haga, M.-a.; van der Molen, S. J., Humidity-Controlled Rectification Switching in Ruthenium-

23 Complex Molecular Junctions. Nature Nanotechnology 2018, 13, 117-121. 
36. Chen, X.; Roemer, M.; Yuan, L.; Du, W.; Thompson, D.; del Barco, E.; Nijhuis, C. A.,

Molecular Diodes with Rectification Ratios Exceeding 105 Driven by Electrostatic Interactions.

Nature Nanotechnology 2017, 12, 797.

37. Yuan, L.; Nerngchamnong, N.; Cao, L.; Hamoudi, H.; del Barco, E.; Roemer, M.; Sriramula,

R. K.; Thompson, D.; Nijhuis, C. A., Controlling the Direction of Rectification in a Molecular

Diode. Nature Communications 2015, 6, 6324.

7 38. Zhang, G.-P.; Wang, S.; Wei, M.-Z.; Hu, G.-C.; Wang, C.-K., Tuning the Direction of

8 Rectification by Adjusting the Location of the Bipyridyl Group in Alkanethiolate Molecular

9 Diodes. The Journal of Physical Chemistry C 2017, 121, 7643-7648.

10 39. Duche, D.; Planchoke, U.; Dang, F.-X.; Rouzo, J. L.; Bescond, M.; Simon, J.-J.; Balaban, T.

11 S.; Escoubas, L., Model of Self Assembled Monolayer Based Molecular Diodes Made of

12 Ferrocenyl-Alkanethiols. Journal of Applied Physics 2017, 121, 115503.

13 40. Nguyen, Q. v.; Martin, P.; Frath, D.; Della Rocca, M. L.; Lafolet, F.; Barraud, C.; Lafarge,

14 P.; Mukundan, V.; James, D.; McCreery, R. L.; Lacroix, J.-C., Control of Rectification in

15 Molecular Junctions: Contact Effects and Molecular Signature. Journal of the American

16

17

18

19

20
Chemical Society 2017, 139, 11913-11922.

41. Fahlman, M.; Crispin, A.; Crispin, X.; Henze, S.; De Jong, M.; Osikowicz, W.; Tengstedt, C.; Salaneck, W., Electronic Structure of Hybrid Interfaces for Polymer-Based Electronics. Journal of physics. Condensed matter : an Institute of Physics journal 2007, 19, 183202.

42. Otero, R.; Vázquez de Parga, A. L.; Gallego, J. M., Electronic, Structural and Chemical Effects of Charge-Transfer at Organic/Inorganic Interfaces. Surface Science Reports 2017, 72, 105-145. 
43. Vilan, A., (Invited) Energy Alignment at the Molecule - Electrode Interface: An

2 Electrochemical-Potential / Hardness View. ECS Transactions 2018, 86, 67-78.

3 44. Haag, R.; Rampi, M. A.; Holmlin, R. E.; Whitesides, G. M., Electrical Breakdown of

4 Aliphatic and Aromatic Self-Assembled Monolayers Used as Nanometer-Thick Organic

5 Dielectrics. Journal of the American Chemical Society 1999, 121, 7895-7906.

6 45. Yuan, L.; Jiang, L.; Nijhuis, C. A., The Drive Force of Electrical Breakdown of Large-Area

7 Molecular Tunnel Junctions. Advanced Functional Materials 2018, 28, 1801710.

8 46. Thompson, D.; Liao, J.; Nolan, M.; Quinn, A. J.; Nijhuis, C. A.; O’Dwyer, C.; Nirmalraj, P.

9 N.; Schönenberger, C.; Calame, M., Formation Mechanism of Metal-Molecule-Metal Junctions:

10 Molecule-Assisted Migration on Metal Defects. The Journal of Physical Chemistry C 2015, 119,

$11 \quad 19438-19451$.

12 47. Katsouras, I.; Kronemeijer, A. J.; Smits, E. C. P.; Hal, P. A. v.; Geuns, T. C. T.; Blom, P. W.

13 M.; Leeuw, D. M. d., Extending the Voltage Window in the Characterization of Electrical

14 Transport of Large-Area Molecular Junctions. Applied Physics Letters 2011, 99, 013303.

15 48. Van Vooren, A.; Lemaur, V.; Ye, A.; Beljonne, D.; Cornil, J., Impact of Bridging Units on the Dynamics of Photoinduced Charge Generation and Charge Recombination in DonorAcceptor Dyads. ChemPhysChem 2007, 8, 1240-1249.

49. Joachim, C.; Ratner, M. A., Molecular Electronics: Some Views on Transport Junctions and Beyond. Proceedings of the National Academy of Sciences of the United States of America 2005, 102, 8801-8808.

50. Paulson, B. P.; Miller, J. R.; Gan, W.-X.; Closs, G., Superexchange and Sequential Mechanisms in Charge Transfer with a Mediating State between the Donor and Acceptor. Journal of the American Chemical Society 2005, 127, 4860-4868. 
Topography of the Bottom Electrodes in Blocking Leakage Currents in Molecular Diodes.

Journal of the American Chemical Society 2014, 136, 6554-6557.

52. Jiang, L.; Sangeeth, C. S. S.; Wan, A.; Vilan, A.; Nijhuis, C. A., Defect Scaling with Contact Area in Egain-Based Junctions: Impact on Quality, Joule Heating, and Apparent Injection Current. The Journal of Physical Chemistry C 2015, 119, 960-969. Veciana, J., Influence of the Donor Unit on the Rectification Ratio in Tunnel Junctions Based on Donor-Acceptor Sams Using Ptm Units as Acceptors. Physical Chemistry Chemical Physics 2018, 20, 25638-25647.

11 54. Jiang, L.; Yuan, L.; Cao, L.; Nijhuis, C. A., Controlling Leakage Currents: The Role of the

12 Binding Group and Purity of the Precursors for Self-Assembled Monolayers in the Performance

13 of Molecular Diodes. Journal of the American Chemical Society 2014, 136, 1982-1991.

14 55. Cometto, F. P.; Ruano, G.; Ascolani, H.; Zampieri, G., Adlayers of Alkanedithiols on

15 Au(111): Effect of Disulfide Reducing Agent. Langmuir 2013, 29, 1400-1406.

16 56. Yuan, L.; Thompson, D.; Cao, L.; Nerngchangnong, N.; Nijhuis, C. A., One Carbon Matters:

17 The Origin and Reversal of Odd-Even Effects in Molecular Diodes with Self-Assembled

18 Monolayers of Ferrocenyl-Alkanethiolates. The Journal of Physical Chemistry C 2015, 119,

$19 \quad 17910-17919$.

20 57. Toledano, T.; Sazan, H.; Mukhopadhyay, S.; Alon, H.; Lerman, K.; Bendikov, T.; Major, D.

21 T.; Sukenik, C. N.; Vilan, A.; Cahen, D., Odd-Even Effect in Molecular Electronic Transport

22 Via an Aromatic Ring. Langmuir 2014, 30, 13596-13605. 
58. Gnatek, D.; Schuster, S.; Ossowski, J.; Khan, M.; Rysz, J.; Krakert, S.; Terfort, A.;

Zharnikov, M.; Cyganik, P., Odd-Even Effects in the Structure and Stability of Azobenzene-

Substituted Alkanethiolates on Au(111) and Ag(111) Substrates. The Journal of Physical

Chemistry C 2015, 119, 25929-25944.

59. Chen, J.; Chang, B.; Oyola-Reynoso, S.; Wang, Z.; Thuo, M., Quantifying Gauche Defects and Phase Evolution in Self-Assembled Monolayers through Sessile Drops. ACS Omega 2017, 2,

$7 \quad 2072-2084$.

60. Simão, C.; Mas-Torrent, M.; Casado-Montenegro, J.; Otón, F.; Veciana, J.; Rovira, C., A

9 Three-State Surface-Confined Molecular Switch with Multiple Channel Outputs. Journal of the

10 American Chemical Society 2011, 133, 13256-13259.

11 61. Marchante, E.; Maglione, M. S.; Crivillers, N.; Rovira, C.; Mas-Torrent, M., A Four-State

12 Capacitance Molecular Switch Based on a Redox Active Tetrathiafulvalene Self-Assembled

13 Monolayer. RSC Advances 2017, 7, 5636-5641.

14 62. Bard, A. J. F., L. R, Electrochemical Methods: Fundamentals and Applications. John Wiley

15 \& Sons New York, 2001.

16 63. Yokota, Y.; Miyazaki, A.; Fukui, K.-i.; Enoki, T.; Tamada, K.; Hara, M., Dynamic and

17 Collective Electrochemical Responses of Tetrathiafulvalene Derivative Self-Assembled

18 Monolayers. The Journal of Physical Chemistry B 2006, 110, 20401-20408.

19 64. Kattel, B.; Wang, T.; Kafle, T. R.; Chan, W.-L., The Thickness of the Two-Dimensional

20 Charge Transfer State at the Ttf-Tcnq Interface. Organic Electronics 2017, 48, 371-376.

21 65. Wang, Z.; Dong, H.; Li, T.; Hviid, R.; Zou, Y.; Wei, Z.; Fu, X.; Wang, E.; Zhen, Y.;

22 Nørgaard, K.; Laursen, B. W.; Hu, W., Role of Redox Centre in Charge Transport Investigated 
$2 \quad 2015,6,7478$.

3 66. Reus, W. F.; Nijhuis, C. A.; Barber, J. R.; Thuo, M. M.; Tricard, S.; Whitesides, G. M.,

4 Statistical Tools for Analyzing Measurements of Charge Transport. The Journal of Physical

$5 \quad$ Chemistry C 2012, 116, 6714-6733.

6 67. Love, J. C.; Estroff, L. A.; Kriebel, J. K.; Nuzzo, R. G.; Whitesides, G. M., Self-Assembled

7 Monolayers of Thiolates on Metals as a Form of Nanotechnology. Chemical Reviews 2005, 105,

$8 \quad 1103-1170$.

9 68. Xie, Z.; Bâldea, I.; Smith, C. E.; Wu, Y.; Frisbie, C. D., Experimental and Theoretical

10 Analysis of Nanotransport in Oligophenylene Dithiol Junctions as a Function of Molecular

11 Length and Contact Work Function. ACS Nano 2015, 9, 8022-8036.

12 69. Heimel, G.; Romaner, L.; Zojer, E.; Brédas, J.-L., Toward Control of the Metal-Organic 13 Interfacial Electronic Structure in Molecular Electronics: A First-Principles Study on Self-

14 Assembled Monolayers of П-Conjugated Molecules on Noble Metals. Nano Letters 2007, 7, $15 \quad 932-940$.

70. Smith, C. E.; Xie, Z.; Bâldea, I.; Frisbie, C. D., Work Function and Temperature

17 Dependence of Electron Tunneling through an N-Type Perylene Diimide Molecular Junction

18 with Isocyanide Surface Linkers. Nanoscale 2018, 10, 964-975.

19 71. Fonseca Guerra, C.; Handgraaf, J.-W.; Baerends, E. J.; Bickelhaupt, F. M., Voronoi

20 Deformation Density (Vdd) Charges: Assessment of the Mulliken, Bader, Hirshfeld, Weinhold,

21 and Vdd Methods for Charge Analysis. Journal of Computational Chemistry 2004, 25, 189-210.

22 72. Diez-Cabanes, V.; Gonzalez, S. R.; Osella, S.; Cornil, D.; Van Dyck, C.; Cornil, J., Energy

23 Level Alignment at Interfaces between Au (111) and Thiolated Oligophenylenes of Increasing 
Chain Size: Theoretical Evidence of Pinning Effects. Advanced Theory and Simulations 2018, 1,

21700020.

3 73. Rodriguez-Gonzalez, S.; Xie, Z.; Galangau, O.; Selvanathan, P.; Norel, L.; Van Dyck, C.;

4 Costuas, K.; Frisbie, C. D.; Rigaut, S.; Cornil, J., Homo Level Pinning in Molecular Junctions:

5 Joint Theoretical and Experimental Evidence. The Journal of Physical Chemistry Letters 2018,

$69,2394-2403$.

7 74. Kim, B.; Choi, S. H.; Zhu, X. Y.; Frisbie, C. D., Molecular Tunnel Junctions Based on П-

8 Conjugated Oligoacene Thiols and Dithiols between Ag, Au, and Pt Contacts: Effect of Surface

9 Linking Group and Metal Work Function. Journal of the American Chemical Society 2011, 133,

$10 \quad 19864-19877$.

11 75. Chiechi, R. C.; Weiss, E. A.; Dickey, M. D.; Whitesides, G. M., Eutectic Gallium-Indium

12 (Egain): A Moldable Liquid Metal for Electrical Characterization of Self-Assembled

13 Monolayers. Angewandte Chemie International Edition 2008, 47, 142-144.

14 76. Han, Y. N., C.; Maglione, M. S.; Karuppannan, S. K.; Casado-Montenegro, J.; Qi, D.C.;

15 Chen, X. P.; Tadich, A.; Cowie, B.; Mas-Torrent, M.; Rovira, C.; Cornil, J.; Veciana, J.; Barco,

E. D.; and Nijhuis, C. A, Bias-Polarity Dependent Direct and Inverted Marcus Charge Transport

17 Affecting Rectification in a Redox-Active Molecular Junction. Manuscript Submitted.

18 77. Yuan, L.; Breuer, R.; Jiang, L.; Schmittel, M.; Nijhuis, C. A., A Molecular Diode with a

19 Statistically Robust Rectification Ratio of Three Orders of Magnitude. Nano Letters 2015, 15,

$20 \quad 5506-5512$.

21 78. Vilan, A., Revealing Tunnelling Details by Normalized Differential Conductance Analysis

22 of Transport across Molecular Junctions. Physical Chemistry Chemical Physics 2017, 19, 27166-

$23 \quad 27172$ 
2 Tunneling Model: Scaling and Linearization. The Journal of Physical Chemistry C 2007, 111,

3 4431-4444.

4 80. Karuppannan, S. K.; Hongting, H.; Troadec, C.; Vilan, A.; Nijhuis, C. A., Ultrasmooth and

5 Photoresist-Free Micropore-Based Egain Molecular Junctions: Fabrication and How Roughness

6 Determines Voltage Response. Advanced Functional Materials 2019, 29, 1904452.

7 81. Fereiro, J. A.; Yu, X.; Pecht, I.; Sheves, M.; Cuevas, J. C.; Cahen, D., Tunneling Explains

8 Efficient Electron Transport Via Protein Junctions. Proceedings of the National Academy of

$9 \quad$ Sciences 2018, 115, E4577.

10 82. Hayakawa, R.; Hiroshiba, N.; Chikyow, T.; Wakayama, Y., Single-Electron Tunneling

11 through Molecular Quantum Dots in a Metal-Insulator-Semiconductor Structure. Adv. Funct.

$12 \quad$ Mater 2011, 21, 2933-2937.

13 83. Brédas, J.-L.; Beljonne, D.; Coropceanu, V.; Cornil, J., Charge-Transfer and Energy-

14 Transfer Processes in П-Conjugated Oligomers and Polymers: A Molecular Picture. Chemical

15 Reviews 2004, 104, 4971-5004.

84. Coropceanu, V.; Cornil, J.; da Silva Filho, D. A.; Olivier, Y.; Silbey, R.; Brédas, J.-L.,

17 Charge Transport in Organic Semiconductors. Chemical Reviews 2007, 107, 926-952. 